CRYSTALLOGRAPHIC COMMUNICATIONS

ISSN 2056-9890

Received 9 January 2020

Accepted 25 January 2020

Edited by A. J. Lough, University of Toronto, Canada

Keywords: crystal structure; 1,4-benzothiazin-3one; dihydrothiazine; hydrogen bond; $\pi$ stacking; Hirshfeld surface.

CCDC reference: 1980073

Supporting information: this article has supporting information at journals.iucr.org/e

\section{Crystal structure, Hirshfeld surface analysis, interaction energy and DFT studies of (2Z)-2-(2,4- dichlorobenzylidene)-4-nonyl-3,4-dihydro-2H-1,4- benzothiazin-3-one}

\author{
Brahim Hni, ${ }^{a}$ Nada Kheira Sebbar, ${ }^{b, a}$ Tuncer Hökelek, ${ }^{c}$ Achour Redouane, ${ }^{a}$ Joel T. \\ Mague, $^{\mathrm{d}}$ Noureddine Hamou Ahabchane ${ }^{\mathrm{a}}$ and El Mokhtar Essassi ${ }^{\mathrm{a}}$
}

\begin{abstract}
a Laboratoire de Chimie Organique Hétérocyclique URAC 21, Pôle de Compétence Pharmacochimie, Av. Ibn Battouta BP 1014, Faculté des Sciences, Université Mohammed V, Rabat, Morocco, 'baboratoire de Chimie Appliquée et Environnement, Equipe de Chimie Bioorganique Appliquée, Faculté des Sciences, Université lbn Zohr, Agadir, Morocco, ${ }^{\mathbf{c}}$ Department of Physics, Hacettepe University, 06800 Beytepe, Ankara, Turkey, and ${ }^{\mathbf{d}}$ Department of Chemistry, Tulane University, New Orleans, LA 70118, USA. *Correspondence e-mail: brahimhni2018@gmail.com
\end{abstract}

The title compound, $\mathrm{C}_{24} \mathrm{H}_{27} \mathrm{Cl}_{2} \mathrm{NOS}$, contains 1,4-benzothiazine and 2,4dichlorophenylmethylidene units in which the dihydrothiazine ring adopts a screw-boat conformation. In the crystal, intermolecular $\mathrm{C}-\mathrm{H}_{\mathrm{Bnz}} \cdots \mathrm{O}_{\mathrm{Thz}}(\mathrm{Bnz}=$ benzene and $\mathrm{Thz}=$ thiazine) hydrogen bonds form chains of molecules extending along the $a$-axis direction, which are connected to their inversionrelated counterparts by $\mathrm{C}-\mathrm{H}_{\mathrm{Bnz}} \cdots \mathrm{Cl}_{\text {Dchlphy }}$ (Dchlphy $=2$,4-dichlorophenyl) hydrogen bonds and $\mathrm{C}-\mathrm{H}_{\text {Dchlphy }} \cdots \pi$ (ring) interactions. These double chains are further linked by $\mathrm{C}-\mathrm{H}_{\text {Dchlphy }} \cdots \mathrm{O}_{\mathrm{Thz}}$ hydrogen bonds, forming stepped layers approximately parallel to (012). The Hirshfeld surface analysis of the crystal structure indicates that the most important contributions for the crystal packing are from $\mathrm{H} \cdots \mathrm{H}(44.7 \%), \mathrm{C} \cdots \mathrm{H} / \mathrm{H} \cdots \mathrm{C}(23.7 \%), \mathrm{Cl} \cdot \mathrm{H} / \mathrm{H} \cdots \mathrm{Cl}$ $(18.9 \%), \quad \mathrm{O} \cdots \mathrm{H} / \mathrm{H} \cdots \mathrm{O} \quad(5.0 \%)$ and $\mathrm{S} \cdots \mathrm{H} / \mathrm{H} \cdots \mathrm{S} \quad(4.8 \%)$ interactions. Hydrogen-bonding and van der Waals interactions are the dominant interactions in the crystal packing. Computational chemistry indicates that in the crystal, $\mathrm{C}-\mathrm{H}_{\text {Dchlphy }} \cdots \mathrm{O}_{\mathrm{Thz}}, \mathrm{C}-\mathrm{H}_{\mathrm{Bnz}} \cdots \mathrm{O}_{\mathrm{Thz}}$ and $\mathrm{C}-\mathrm{H}_{\mathrm{Bnz}} \cdots \mathrm{Cl}_{\text {Dchlphy }}$ hydrogenbond energies are 134.3, 71.2 and $34.4 \mathrm{~kJ} \mathrm{~mol}^{-1}$, respectively. Density functional theory (DFT) optimized structures at the B3LYP/6-311 G(d,p) level are compared with the experimentally determined molecular structure in the solid state. The HOMO-LUMO behaviour was elucidated to determine the energy gap. The two carbon atoms at the end of the nonyl chain are disordered in a $0.562(4) / 0.438$ (4) ratio.

\section{Chemical context}

A number of sulfur- and nitrogen-containing heterocyclic compounds have been well studied. These molecules exhibit a wide range of biological applications, indicating that the 1,4benzothiazine moiety is a potentially useful template in medicinal chemistry research with therapeutic applications in the antimicrobial (Armenise et al., 2012, Sabatini et al., 2008), anti-viral (Malagu et al., 1998), anti-oxidant (Zia-ur-Rehman et al. 2009), anti-inflammatory (Trapani et al., 1985; Gowda et al., 2011) antipyretic (Warren et al., 1987), and anti-cancer (Gupta et al., 1991; Gupta et al., 1985) areas as well as being precursors for the synthesis of new compounds (Sebbar et al., 2015a; Vidal et al., 2006) possessing anti-diabetic (Tawada et al., 1990) and anti-corrosion activities (Ellouz et al., 2016a,b; Sebbar et al., 2016a) and biological properties (Hni et al., 
2019a; Ellouz et al., 2017a,b, 2018; Sebbar et al., 2019a,b). As a continuation of our research into the development of new 1,4benzothiazine derivatives with potential pharmacological applications, we have studied the reaction of 1-bromononane with (Z)-2-(2,4-dichlorobenzylidene)-2 $H$-1,4-benzothiazin$3(4 H)$-one under phase-transfer catalysis conditions using tetra- $n$-butylammonium bromide (TBAB) as catalyst and potassium carbonate as base (Hni et al., 2019b; Sebbar et al., 2019) to give the title compound, (I), in good yield. We report here its crystalline and molecular structures as well as the Hirshfeld surface analysis and the density functional theory (DFT) computational calculations.

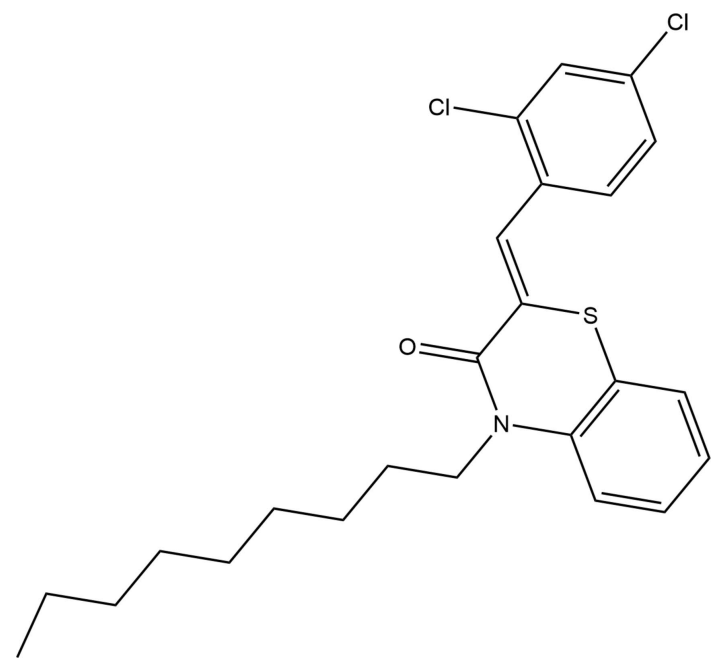

\section{Structural commentary}

The title compound contains 1,4-benzothiazine and 2,4-dichlorophenylmethylidene units (Fig. 1), in which the dihydrothiazine ring, $B(\mathrm{~S} 1 / \mathrm{N} 1 / \mathrm{C} 1 / \mathrm{C} 6-\mathrm{C} 8)$, adopts a screw-boat conformation with puckering parameters $Q_{\mathrm{T}}=0.5581$ (16) $\AA$, $\theta=69.76(18)^{\circ}$ and $\varphi=334.3(2)^{\circ}$. The planar rings, $A(\mathrm{C} 1-\mathrm{C} 6)$ and $C(\mathrm{C} 10-\mathrm{C} 15)$ are oriented at a dihedral angle of $88.45(7)^{\circ}$.

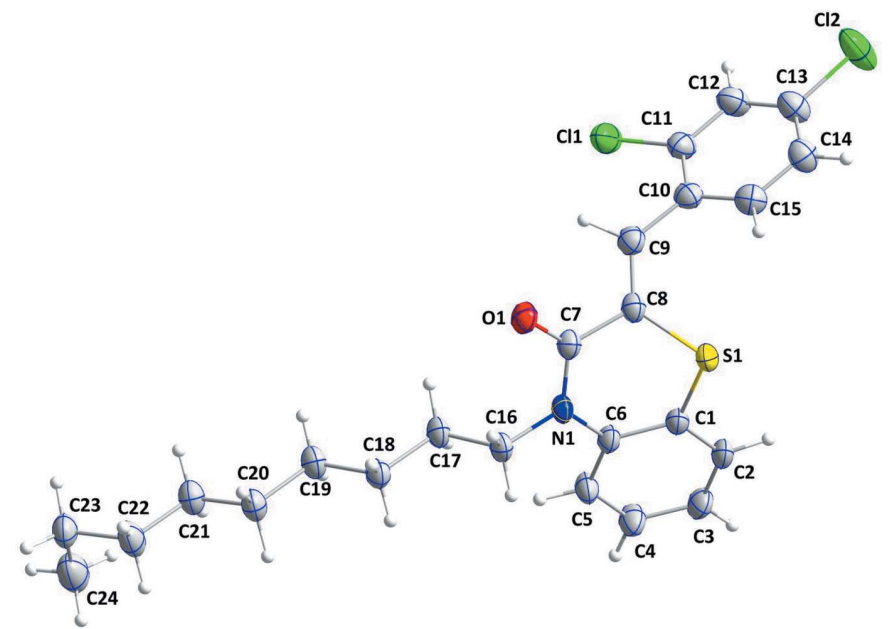

Figure 1

The molecular structure of the title compound with the atom-numbering scheme. Displacement ellipsoids are drawn at the $50 \%$ probability level.

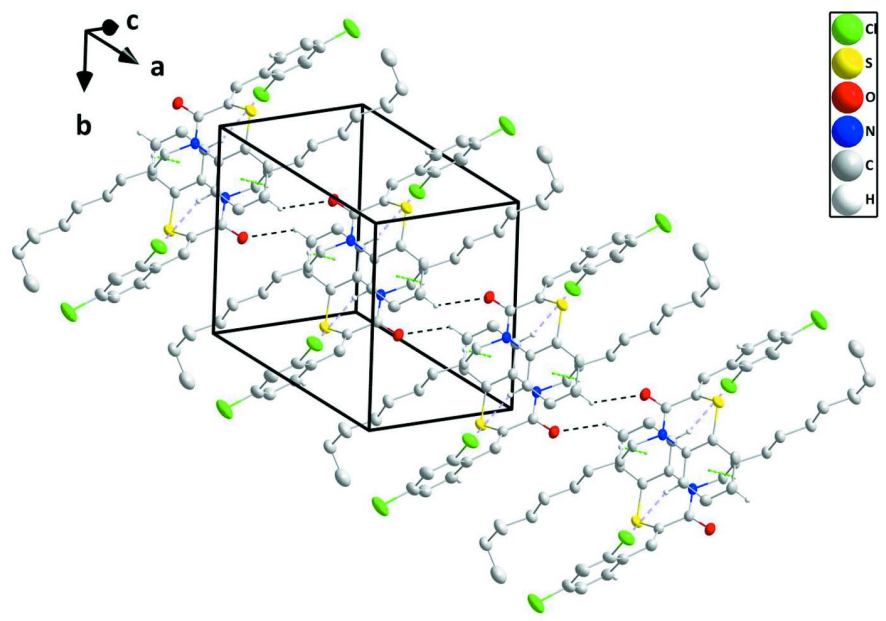

Figure 2

A perspective view of one double chain. The intermolecular $\mathrm{C}-$ $\mathrm{H}_{\mathrm{Bnz}} \cdots \mathrm{O}_{\mathrm{Thz}}$ and $\mathrm{C}-\mathrm{H}_{\mathrm{Bnz}} \cdots \mathrm{Cl}_{\text {Dehlphy }}(\mathrm{Bnz}=$ benzene, Thz $=$ thiazine and Dchlphy = 2,4-dichlorophenyl) hydrogen bonds are shown, respectively, as black and light purple dashed lines while the $\mathrm{C}-\mathrm{H}_{\text {Dchlphy }} \cdots \pi$ (ring) interactions are shown as green dashed lines.

Atoms $\mathrm{Cl} 1, \mathrm{Cl} 2$ and $\mathrm{C} 9$ are almost co-planar with ring $C$ being displaced by $0.0247(6),-0.0732$ (9) and -0.0274 (2) $\AA$, respectively.

\section{Supramolecular features}

In the crystal, $\mathrm{C}-\mathrm{H}_{\mathrm{Bnz}} \cdots \mathrm{O}_{\mathrm{Thz}}(\mathrm{Bnz}=$ benzene and $\mathrm{Thz}=$ thiazine) hydrogen bonds link the molecules, forming chains extending along the $a$-axis direction, which are connected to their inversion-related counterparts by $\mathrm{C}-\mathrm{H}_{\mathrm{Bnz}} \cdots \mathrm{Cl}_{\text {Dchlphy }}$ (Dchlphy $=2$,4-dichlorophenyl) hydrogen bonds and $\mathrm{C}-$ $\mathrm{H}_{\text {Dchlphy }} \cdots \pi$ (ring) interactions (Table 1 and Fig. 2). These double chains are further linked by $\mathrm{C}-\mathrm{H}_{\text {Dchlphy }} \cdots \mathrm{O}_{\text {Thz }}$ hydrogen bonds to form stepped layers approximately parallel to (012) (Table 1 and Figs. 2 and 3).

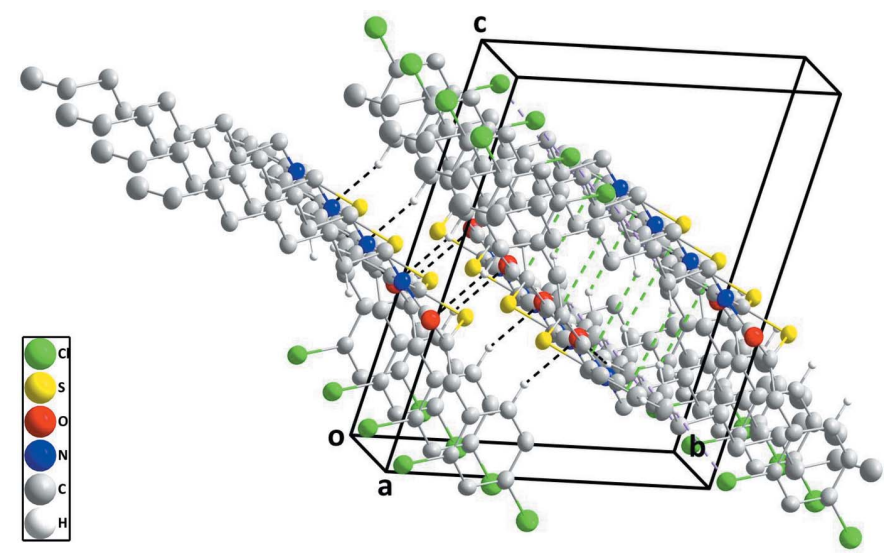

Figure 3

Perspective view of one double chain and half of a second showing the $\mathrm{C}-\mathrm{H}_{\text {Dchlphy }} \cdots \mathrm{O}_{\text {Thz }}$ (Dchlphy $=2,4$-dichlorophenyl and Thz $=$ thiazine $)$ hydrogen bond connecting them. Intermolecular interactions depicted as in Fig. 2. 
Table 1

Hydrogen-bond geometry $\left(\AA,^{\circ}\right)$.

$C g 1$ is the centroid of the ring $A(\mathrm{C} 1-\mathrm{C} 6)$.

\begin{tabular}{lllll}
\hline$D-\mathrm{H} \cdots A$ & $D-\mathrm{H}$ & $\mathrm{H} \cdots A$ & $D \cdots A$ & $D-\mathrm{H} \cdots A$ \\
\hline $\mathrm{C} 3-\mathrm{H} 3 \cdots \mathrm{O} 1^{\mathrm{ix}}$ & $0.96(3)$ & $2.51(3)$ & $3.268(2)$ & $136(2)$ \\
$\mathrm{C} 5-\mathrm{H} 5 \cdots \mathrm{Cl}^{\mathrm{i}}$ & $0.96(2)$ & $2.86(2)$ & $3.634(2)$ & $138.8(17)$ \\
$\mathrm{C} 15-\mathrm{H} 15 \cdots \mathrm{O} 1^{\mathrm{vi}}$ & $0.96(3)$ & $2.36(3)$ & $3.270(2)$ & $159(2)$ \\
$\mathrm{C} 17-\mathrm{H} 17 A \cdots C g 1^{\mathrm{i}}$ & $0.98(2)$ & $2.90(2)$ & $3.619(2)$ & $131.2(17)$ \\
\hline
\end{tabular}

Symmetry codes: (i) $-x+1,-y+1,-z+1$; (vi) $-x+1,-y,-z+1$; (ix) $x+1, y, z$.

\section{Hirshfeld surface analysis}

In order to visualize the intermolecular interactions in the crystal of the title compound, a Hirshfeld surface (HS) analysis (Hirshfeld, 1977; Spackman \& Jayatilaka, 2009) was carried out by using Crystal Explorer 17.5 (Turner et al., 2017). In the HS plotted over $d_{\text {norm }}$ (Fig. 4), the white surface indicates contacts with distances equal to the sum of van der Waals radii, and the red and blue colours indicate distances shorter (in close contact) or longer (distinct contact) than the van der Waals radii (Venkatesan et al., 2016). The bright-red spots appearing near $\mathrm{O} 1$ and hydrogen atom $\mathrm{H} 15$ indicate their roles as the respective donors and/or acceptors; they also appear as blue and red regions corresponding to positive and negative potentials on the HS mapped over electrostatic potential (Spackman et al., 2008; Jayatilaka et al., 2005) as shown in Fig. 5. The blue regions indicate positive electrostatic potential (hydrogen-bond donors), while the red regions indicate negative electrostatic potential (hydrogen-bond acceptors). The shape-index of the HS is a tool to visualize $\pi-$ $\pi$ stacking by the presence of adjacent red and blue triangles; if there are no adjacent red and/or blue triangles, then there are no $\pi-\pi$ interactions. Fig. 6 clearly suggests that there are no $\pi-\pi$ interactions in (I). The overall two-dimensional fingerprint plot, Fig. $7 a$, and those delineated into $\mathrm{H} \cdots \mathrm{H}$, $\mathrm{C} \cdots \mathrm{H} / \mathrm{H} \cdots \mathrm{C}, \mathrm{Cl} \cdots \mathrm{H} / \mathrm{H} \cdots \mathrm{Cl}, \mathrm{O} \cdots \mathrm{H} / \mathrm{H} \cdots \mathrm{O}$ and $\mathrm{S} \cdots \mathrm{H} /$ $\mathrm{H}$...S contacts (McKinnon et al., 2007) are illustrated in Fig. $7 b-f$, respectively, together with their relative contributions to the Hirshfeld surface. The most important interaction is $\mathrm{H} \cdots \mathrm{H}$ (Table 2), contributing $44.7 \%$ to the overall crystal

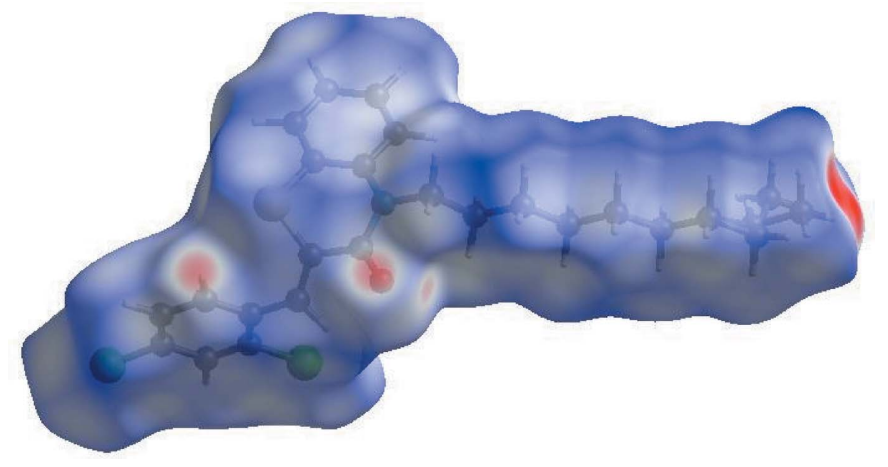

Figure 4

View of the three-dimensional Hirshfeld surface of the title compound plotted over $d_{\text {norm }}$ in the range -0.6343 to 1.4076 a.u.

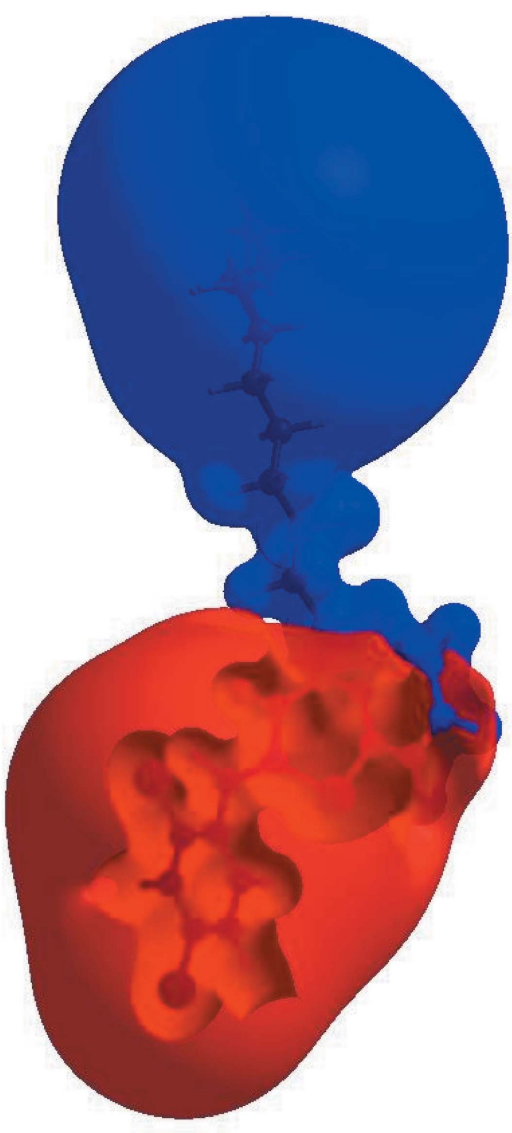

Figure 5

View of the three-dimensional Hirshfeld surface of the title compound plotted over electrostatic potential energy in the range -0.0500 to 0.0500 a.u. using the STO-3 G basis set at the Hartree-Fock level of theory. Hydrogen-bond donors and acceptors are shown as blue and red regions around the atoms corresponding to positive and negative potentials, respectively.

packing, which is reflected in Fig. $7 b$ as widely scattered points of high density due to the large hydrogen content of the molecule with the tip at $d_{\mathrm{e}}=d_{\mathrm{i}}=1.09 \AA$ A. The presence of C$\mathrm{H} \cdots \pi$ interactions is indicated by the fringed pairs of characteristic wings in the fingerprint plot delineated into $\mathrm{C} \cdot \mathrm{H} /$ H. . C contacts (Fig. $7 c, 23.7 \%$ contribution to the HS). The two pairs of wings in the fingerprint plot delineated into

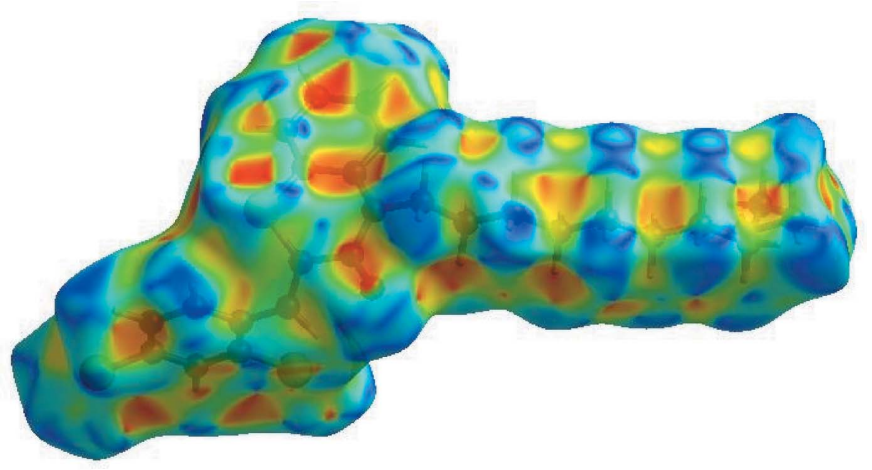

Figure 6

Hirshfeld surface of the title compound plotted over shape-index. 

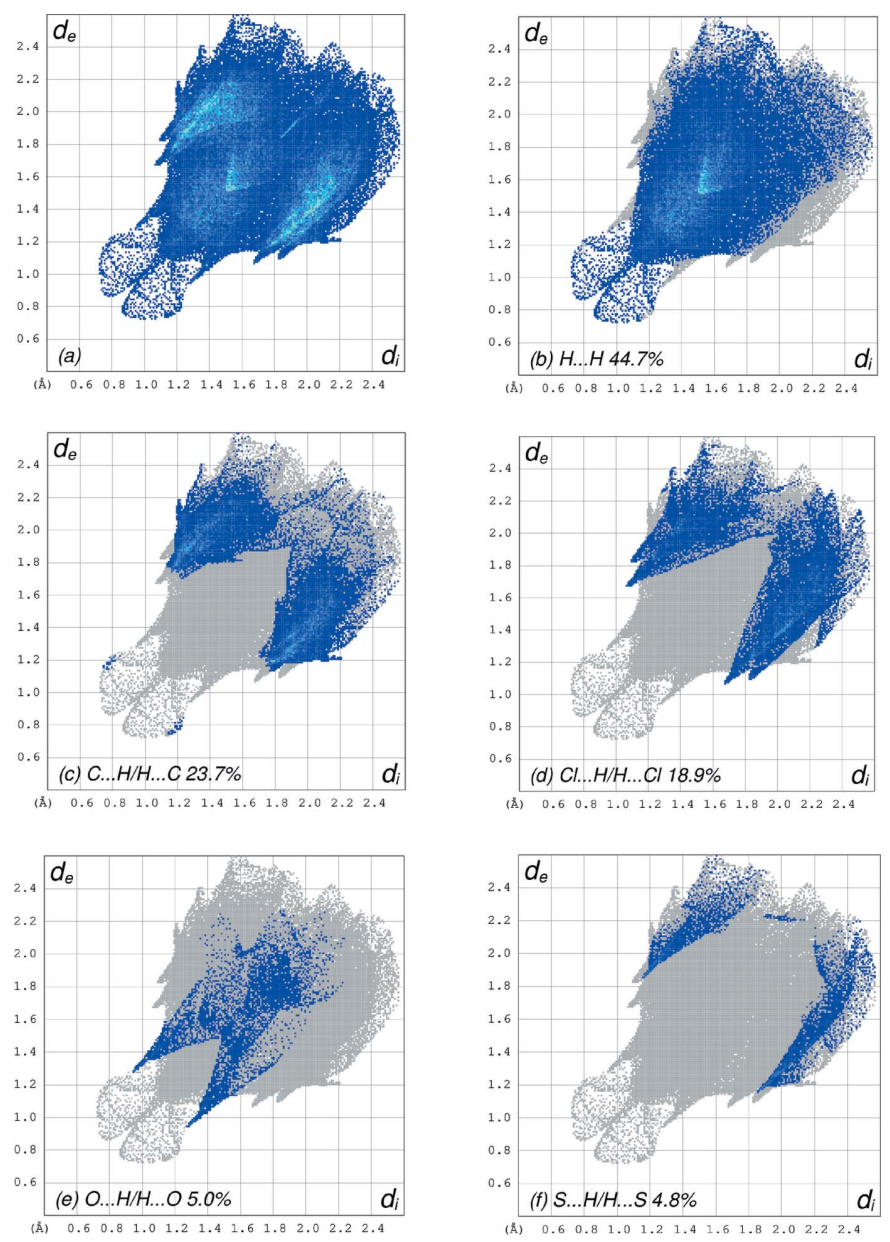

Table 2

Selected interatomic distances $(\AA)$.

\begin{tabular}{|c|c|c|c|}
\hline $\mathrm{Cl} 1 \cdots \mathrm{C} 5^{\mathrm{i}}$ & $3.634(2)$ & $\mathrm{C} 21 \cdots \mathrm{H} 24 B$ & 2.86 \\
\hline $\mathrm{Cl} 1 \cdots \mathrm{C} 12^{\mathrm{ii}}$ & $3.548(2)$ & $\mathrm{C} 24 \cdots \mathrm{H} 21 B$ & 2.91 \\
\hline $\mathrm{Cl} 1 \cdots \mathrm{H} 9$ & $2.82(3)$ & $\mathrm{C} 24 A \cdots \mathrm{H} 24 E^{\mathrm{viii}}$ & 2.44 \\
\hline $\mathrm{Cl} 1 \cdots \mathrm{H} 5^{\mathrm{i}}$ & $2.86(2)$ & $\mathrm{C} 24 A \cdots \mathrm{H} 24 F^{\mathrm{viii}}$ & 2.70 \\
\hline $\mathrm{Cl} 1 \cdots \mathrm{H} 12^{\mathrm{ii}}$ & $2.92(3)$ & $\mathrm{C} 24 A \cdots \mathrm{H} 24 D^{\text {viii }}$ & 1.94 \\
\hline $\mathrm{Cl} 2 \cdots \mathrm{H} 20 A^{\mathrm{iii}}$ & $3.13(2)$ & $\mathrm{H} 3 \cdots \mathrm{H} 17 A^{\mathrm{ix}}$ & $2.42(4)$ \\
\hline $\mathrm{Cl} 2 \cdots \mathrm{H} 24 C^{\mathrm{i}}$ & 3.01 & $\mathrm{H} 5 \cdots \mathrm{H} 17 B$ & $2.21(4)$ \\
\hline $\mathrm{S} 1 \cdots \mathrm{N} 1$ & 3.0439 (16) & $\mathrm{H} 5 \cdots \mathrm{H} 16 B$ & $2.33(3)$ \\
\hline $\mathrm{S} 1 \cdots \mathrm{C} 15$ & $3.236(2)$ & $\mathrm{H} 12 \cdots \mathrm{H} 22 A^{\mathrm{iii}}$ & 2.37 \\
\hline $\mathrm{S} 1 \cdots \mathrm{H} 15$ & $2.84(3)$ & $\mathrm{H} 16 A \cdots \mathrm{H} 18 A$ & $2.47(3)$ \\
\hline $\mathrm{S} 1 \cdots \mathrm{H} 2^{\mathrm{iv}}$ & $3.15(3)$ & $\mathrm{H} 16 B \cdots \mathrm{H} 24 D^{\mathrm{vii}}$ & 2.54 \\
\hline $\mathrm{O} 1 \cdots \mathrm{C} 3^{\mathrm{v}}$ & $3.268(2)$ & $\mathrm{H} 16 B \cdots \mathrm{H} 18 B$ & $2.46(3)$ \\
\hline $\mathrm{O} 1 \cdots \mathrm{C} 17$ & $3.238(2)$ & $\mathrm{H} 16 B \cdots \mathrm{H} 24 A^{\text {vii }}$ & 2.49 \\
\hline $\mathrm{O} 1 \cdots \mathrm{C} 15^{\mathrm{vi}}$ & $3.270(2)$ & $\mathrm{H} 17 A \cdots \mathrm{H} 19 A$ & $2.59(3)$ \\
\hline $\mathrm{O} 1 \cdots \mathrm{H}^{\mathrm{v}}$ & $2.51(3)$ & $\mathrm{H} 17 B \cdots \mathrm{H} 19 B$ & $2.55(4)$ \\
\hline $\mathrm{O} 1 \cdots \mathrm{H} 16 A$ & $2.43(2)$ & $\mathrm{H} 18 B \cdots \mathrm{H} 20 B$ & $2.55(3)$ \\
\hline $\mathrm{O} 1 \cdots \mathrm{H} 17 A$ & $2.75(2)$ & $\mathrm{H} 19 A \cdots \mathrm{H} 21 A$ & $2.58(4)$ \\
\hline $\mathrm{O} 1 \cdots \mathrm{H} 9$ & $2.49(3)$ & $\mathrm{H} 19 B \cdots \mathrm{H} 21 B$ & $2.51(4)$ \\
\hline $\mathrm{O} 1 \cdots \mathrm{H} 15^{\mathrm{vi}}$ & $2.36(3)$ & $\mathrm{H} 20 A \cdots \mathrm{H} 22 A$ & 2.49 \\
\hline $\mathrm{C} 5 \cdots \mathrm{C} 17$ & $3.430(3)$ & $\mathrm{H} 20 B \cdots \mathrm{H} 22 B$ & 2.54 \\
\hline $\mathrm{C} 5 \cdots \mathrm{C} 24^{\mathrm{vii}}$ & 3.58 & $\mathrm{H} 21 A \cdots \mathrm{H} 23 B$ & 2.55 \\
\hline C6 $\cdots C 24^{\mathrm{vii}}$ & 3.58 & $\mathrm{H} 21 A \cdots \mathrm{H} 23 C$ & 2.60 \\
\hline $\mathrm{C} 24 A \cdots \mathrm{C} 24 A^{\text {viii }}$ & 2.48 & $\mathrm{H} 21 B \cdots \mathrm{H} 24 B$ & 2.32 \\
\hline $\mathrm{C} 2 \cdots \mathrm{H} 19 A^{\mathrm{i}}$ & $2.98(2)$ & $\mathrm{H} 21 B \cdots \mathrm{H} 23 D$ & 2.34 \\
\hline $\mathrm{C} 5 \cdots \mathrm{H} 24 A^{\mathrm{vii}}$ & 2.99 & $\mathrm{H} 22 B \cdots \mathrm{H} 24 C$ & 2.27 \\
\hline C5 $\cdots \mathrm{H} 16 B$ & $2.64(2)$ & $\mathrm{H} 22 B \cdots \mathrm{H} 24 E$ & 2.43 \\
\hline C5 $\cdots \mathrm{H} 17 B$ & $2.93(3)$ & $\mathrm{H} 24 D \cdots \mathrm{C} 24 A^{\text {viii }}$ & 1.94 \\
\hline $\mathrm{C} 7 \cdots \mathrm{H} 15^{\mathrm{vi}}$ & $2.95(3)$ & $\mathrm{H} 24 D \cdots \mathrm{H} 24 D^{\text {viii }}$ & 1.82 \\
\hline $\mathrm{C} 7 \cdots \mathrm{H} 17 A$ & $2.99(2)$ & $\mathrm{H} 24 D \cdots \mathrm{H} 24 E^{\mathrm{viii}}$ & 1.70 \\
\hline $\mathrm{C} 16 \cdots \mathrm{H} 5$ & $2.62(3)$ & $\mathrm{H} 24 D \cdots \mathrm{H} 24 F^{\text {viii }}$ & 2.07 \\
\hline $\mathrm{C} 17 \cdots \mathrm{H} 3^{\mathrm{v}}$ & $2.98(3)$ & $\mathrm{H} 24 E \cdots \mathrm{H} 24 F^{\text {viii }}$ & 2.54 \\
\hline $\mathrm{C} 17 \cdots \mathrm{H} 5$ & $2.82(3)$ & & \\
\hline
\end{tabular}

Symmetry codes: (i) $\quad-x+1,-y+1,-z+1$; $\quad$ (ii) $\quad-x+1,-y,-z+2$; $x+1, y-1, z+1$; (iv) $-x+2,-y,-z+1$; (v) $x-1, y, z$; (vi) $-x+1,-y,-z+1$; (vii) $x+1, y-1, z$; (viii) $-x-1,-y+3,-z$; (ix) $x+1, y, z$.

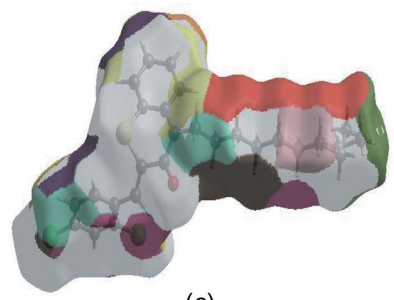

(a)

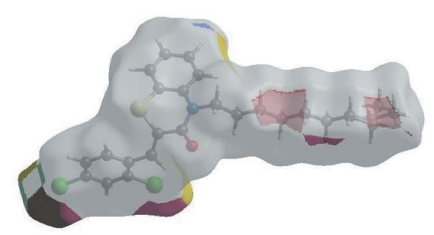

(c)

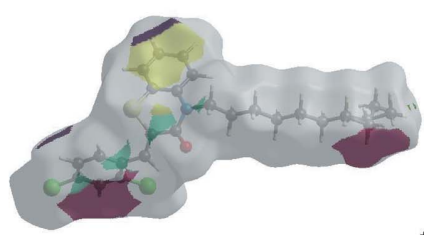

(b)

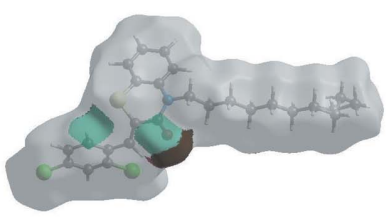

(d) at $d_{\mathrm{e}}+d_{\mathrm{i}}=2.99 \AA$.

The Hirshfeld surface representations with the function $d_{\text {norm }}$ plotted onto the surface are shown for the $\mathrm{H} \cdots \mathrm{H}$, $\mathrm{C} \cdots \mathrm{H} / \mathrm{H} \cdots \mathrm{C}, \mathrm{Cl} \cdots \mathrm{H} / \mathrm{H} \cdots \mathrm{Cl}, \mathrm{O} \cdots \mathrm{H} / \mathrm{H} \cdots \mathrm{O}$ and $\mathrm{S} \cdots \mathrm{H} / \mathrm{H} \cdots \mathrm{S}$ interactions in Fig. $8 a-e$, respectively.

The Hirshfeld surface analysis confirms the importance of $\mathrm{H}$-atom contacts in establishing the packing. The large number of $\mathrm{H} \cdots \mathrm{H}, \mathrm{C} \cdots \mathrm{H} / \mathrm{H} \cdots \mathrm{C}, \mathrm{Cl} \cdots \mathrm{H} / \mathrm{H} \cdots \mathrm{Cl}$ and $\mathrm{O} \cdots \mathrm{H} / \mathrm{H} \cdots \mathrm{O}$ interactions suggest that van der Waals interactions and hydrogen bonding play the major roles in the crystal packing (Hathwar et al., 2015).

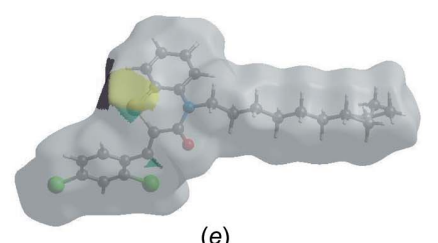

(e)

Figure 8

The Hirshfeld surface representations with the function $d_{\text {norm }}$ plotted onto the surface for $(a) \mathrm{H} \cdots \mathrm{H},(b) \mathrm{C} \cdots \mathrm{H} / \mathrm{H} \cdots \mathrm{C},(c) \mathrm{Cl} \cdots \mathrm{H} / \mathrm{H} \cdots \mathrm{Cl},(d)$ $\mathrm{O} \cdots \mathrm{H} / \mathrm{H} \cdots \mathrm{O}$ and $(f) \mathrm{S} \cdots \mathrm{H} / \mathrm{H} \cdots \mathrm{S}$ interactions. 
Table 3

Comparison of the selected (X-ray and DFT) geometric data $\left(\AA{ }^{\circ}\right)$.

\begin{tabular}{lll}
\hline Bonds/angles & X-ray & B3LYP/6-311G(d,p) \\
\hline Cl1-C11 & $1.744(2)$ & 1.826 \\
C12-C13 & $1.733(2)$ & 1.821 \\
S1-C8 & $1.7578(18)$ & 1.831 \\
S1-C1 & $1.7589(18)$ & 1.830 \\
O1-C7 & $1.228(2)$ & 1.256 \\
N1-C7 & $1.368(2)$ & 1.392 \\
N1-C6 & $1.420(2)$ & 1.423 \\
N1-C16 & $1.479(2)$ & 1.489 \\
& & \\
C8-S1-C1 & $97.27(8)$ & 124.78 \\
C7-N1-C6 & $123.67(14)$ & 114.70 \\
C7-N1-C16 & $117.19(14)$ & 119.29 \\
C6-N1-C16 & $119.07(15)$ & 121.21 \\
C2-C1-C6 & $120.22(17)$ & 117.28 \\
C2-C1-S1 & $119.25(13)$ & 120.47 \\
C6-C1-S1 & $120.52(13)$ & \\
C3-C2-S1 & $120.53(17)$ &
\end{tabular}

\section{Interaction energy calculations}

The intermolecular interaction energies were calculated using the CE-B3LYP/6-31G(d,p) energy model available in Crystal Explorer 17.5 (Turner et al., 2017), where a cluster of molecules is generated by applying crystallographic symmetry operations with respect to a selected central molecule within the default radius of $3.8 \AA$ (Turner et al., 2014). The total intermolecular energy $\left(E_{\mathrm{tot}}\right)$ is the sum of electrostatic $\left(E_{\text {ele }}\right)$, polarization $\left(E_{\mathrm{pol}}\right)$, dispersion $\left(E_{\mathrm{dis}}\right)$ and exchange-repulsion $\left(E_{\text {rep }}\right)$ energies (Turner et al., 2015) with scale factors of 1.057, $0.740,0.871$ and 0.618, respectively (Mackenzie et al., 2017). Hydrogen-bonding interaction energies (in $\mathrm{kJ} \mathrm{mol}^{-1}$ ) were calculated to be $-53.7\left(E_{\text {ele }}\right),-13.6\left(E_{\mathrm{pol}}\right),-161.9\left(E_{\mathrm{dis}}\right), 119.0$ $\left(E_{\text {rep }}\right)$ and $-134.3\left(E_{\text {tot }}\right)$ for $\mathrm{C}-\mathrm{H}_{\text {Dchlphy }} \cdots \mathrm{O}_{\text {Thz }}, 25.6\left(E_{\text {ele }}\right)$, $-5.7\left(E_{\mathrm{pol}}\right),-62.1\left(E_{\mathrm{dis}}\right), 23.1\left(E_{\mathrm{rep}}\right)$ and $-71.2\left(E_{\mathrm{tot}}\right)$ [or C$\mathrm{H}_{\mathrm{Bnz}} \cdots \mathrm{O}_{\mathrm{Thz}}$ and $-16.0\left(E_{\mathrm{ele}}\right),-8.3\left(E_{\mathrm{pol}}\right),-43.0\left(E_{\mathrm{dis}}\right), 42.2$ $\left(E_{\text {rep }}\right)$ and $-34.4\left(E_{\text {tot }}\right)$ for $\mathrm{C}-\mathrm{H}_{\mathrm{Bnz}} \cdots \mathrm{Cl}_{\text {Dchlphy }}(\mathrm{Bnz}=$ benzene, Thz $=$ thiazine and Dchlphy $=2$,4-dichlorophenyl).

\section{DFT calculations}

The optimized structure of the title compound, (I), in the gas phase was generated theoretically via density functional theory (DFT) using the standard B3LYP functional and 6$311 \mathrm{G}(\mathrm{d}, \mathrm{p})$ basis-set calculations as implemented in GAUSSIAN 09 (Frisch et al., 2009). The theoretical and experimental results are in good agreement (Table 3). The highest-occupied molecular orbital (HOMO), acting as an electron donor, and the lowest-unoccupied molecular orbital (LUMO), acting as an electron acceptor, are very important parameters for quantum chemistry. When the energy gap is small, the molecule is highly polarizable and has high chemical reactivity. The DFT calculations provide some important information on the reactivity and site selectivity of the molecular framework. $E_{\text {Hомо }}$ and $E_{\text {LUMO }}$ clarify the inevitable charge-exchange collaboration inside the studied material, and together with the electronegativity $(\chi)$, hardness $(\eta)$, potential $(\mu)$, electrophilicity $(\omega)$ and softness $(\sigma)$ are recorded in Table 4 . The
Table 4

Calculated energies.

\begin{tabular}{ll}
\hline Molecular Energy (a.u.) $(\mathrm{eV})$ & Compound (I) \\
\hline Total Energy, $T E(\mathrm{eV})$ & -64734 \\
$E_{\text {HOMO }}(\mathrm{eV})$ & -6.9440 \\
$E_{\text {LUMO }}(\mathrm{eV})$ & -0.6941 \\
Energy gap, $\Delta E(\mathrm{eV})$ & 6.2499 \\
Dipole moment, $\mu$ (Debye) & 4.4939 \\
Ionization potential, $I(\mathrm{eV})$ & 6.9440 \\
Electron affinity, $A$ & 0.6941 \\
Electro negativity, $\chi$ & 3.8191 \\
Hardness, $\eta$ & 3.1249 \\
Electrophilicity index, $\omega$ & 2.3337 \\
Softness, $\sigma$ & 0.3200 \\
Fraction of electron transferred, $\Delta N$ & 0.5090
\end{tabular}

significance of $\eta$ and $\sigma$ is to evaluate both the reactivity and stability. The electron transition from the HOMO to the LUMO energy level is shown in Fig. 9. The HOMO and LUMO are localized in the plane extending from the whole (2Z)-2-[(2,4-dichlorophenyl)methylidene]-4-nonyl-3,4-dihydro-2 $H$-1,4-benzothiazin-3-one ring.

\section{Database survey}

A search in the Cambridge Structural Database (Groom et al., 2016; updated to October 2019), for compounds containing the fragment II gave 14 hits.
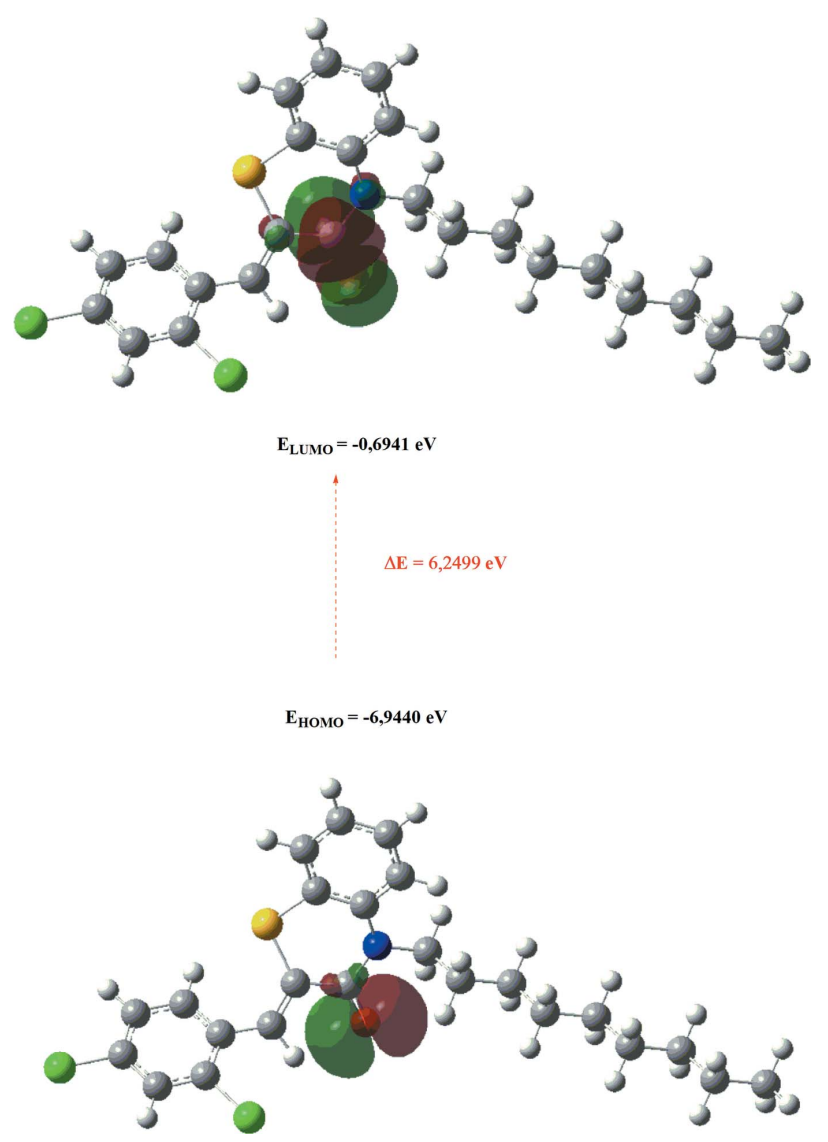

Figure 9

The energy band gap of the title compound. 
<smiles>CN1C(=O)/C(=C/c2ccccc2)Sc2ccccc21</smiles>

II<smiles>O=C(O)CN1C(=O)/C(=C/c2ccccc2)Sc2ccccc21</smiles>

IIb<smiles>O=C1/C(=C/c2ccccc2)Sc2ccccc2N1CC1CC(c2ccccc2)=NO1</smiles>

IId<smiles>C#CCN1C(=O)/C(=C/c2ccccc2)Sc2ccccc21</smiles>

IIa<smiles>O=C1OCCN1CN1C(=O)/C(=C/c2ccccc2)Sc2ccccc21</smiles>

IIc<smiles>[R8]C=C1Sc2ccccc2N([R2])C1=O</smiles>

III
The largest set contains IIa (COGRUN; Sebbar et al., 2014a), IIb (APAJUY; Sebbar et al., 2016c), IIc (EVIYIT; Sebbar et al., 2016b) and IId (WUFGIP; Sebbar et al., 2015b). Additional examples are III: $R_{1}=4-\mathrm{FC}_{6} \mathrm{H}_{4}$ and $R_{2}=$ $\mathrm{CH}_{2} \mathrm{C} \equiv \mathrm{CH}$ (WOCFUS; Hni et al., 2019a), $R_{1}=4-\mathrm{ClC}_{6} \mathrm{H}_{4}$ and $R_{2}=\mathrm{CH}_{2} \mathrm{Ph}$ (OMEGEU; Ellouz et al., 2016c) and $R_{1}=$ 2- $\mathrm{ClC}_{6} \mathrm{H}_{4}, R_{2}=\mathrm{CH}_{2} \mathrm{C} \equiv \mathrm{CH}$ (SAVTUH; Sebbar et al., 2017). In all these compounds, the configuration about the benzylidene group: $\mathrm{C}=\mathrm{CHC}_{6} \mathrm{H}_{5}$ bond is $Z$, and in the majority of these, the heterocyclic ring is quite non-planar with the dihedral angle between the plane defined by the benzene ring plus the nitrogen and sulfur atoms and that defined by nitrogen and sulfur and the other two carbon atoms separating them having approximate values of $36^{\circ}$ (WUFGIP), 29 (APAJUY), $28^{\circ}$ (SAVTUH), $26^{\circ}$ (WOCFUS) and $25^{\circ}$ (COGRUN). By contrast, in both EVIYIT and OMEGEU, the benzothiazine unit is nearly planar with the corresponding dihedral angle being about $4^{\circ}$.

\section{Synthesis and crystallization}

To a solution of (Z)-2-(2,4-dichlorobenzylidene)-2H-1,4benzothiazin-3 $(4 H)$-one $(1.5 \mathrm{mmol})$, potassium carbonate ( $2.7 \mathrm{mmol})$ and tetra- $n$-butyl ammonium bromide $(0.14 \mathrm{mmol})$
Table 5

Experimental details.

Crystal data

Chemical formula

$M_{\mathrm{r}}$

Crystal system, space group

Temperature (K)

$a, b, c(\AA)$

$\alpha, \beta, \gamma\left({ }^{\circ}\right)$

$V\left(\AA^{3}\right)$

Z

Radiation type

$\mu\left(\mathrm{mm}^{-1}\right)$

Crystal size (mm)

$\mathrm{C}_{24} \mathrm{H}_{27} \mathrm{Cl}_{2} \mathrm{NOS}$

448.42

Triclinic, $P \overline{1}$

150

8.9961 (3), 10.3755 (3), 13.2565 (4)

73.857 (1), 88.119 (1), 74.182 (1)

$1142.32(6)$

$\mathrm{Cu} K \alpha$

3.52

$0.20 \times 0.14 \times 0.08$

Data collection

Diffractometer

Bruker D8 VENTURE PHOTON $100 \mathrm{CMOS}$

Absorption correction

$T_{\min }, T_{\max }$

Multi-scan (SADABS; Krause et al., 2015)

No. of measured, independent and observed $[I>2 \sigma(I)]$ reflections

$R_{\text {int }}$

$(\sin \theta / \lambda)_{\max }\left(\AA^{-1}\right)$

$0.54,0.76$

$8788,4246,3772$

0.025

0.618

Refinement

$R\left[F^{2}>2 \sigma\left(F^{2}\right)\right], w R\left(F^{2}\right), S$

No. of reflections

No. of parameters

No. of restraints

$\mathrm{H}$-atom treatment

$\Delta \rho_{\max }, \Delta \rho_{\min }\left(\mathrm{e} \AA^{-3}\right)$

$0.041,0.107,1.02$

4246

349

14

$\mathrm{H}$ atoms treated by a mixture of independent and constrained refinement

$0.32,-0.50$

Computer programs: APEX3 and SAINT (Bruker, 2016), SHELXT (Sheldrick, 2015a), SHELXL2018/1 (Sheldrick, 2015b), DIAMOND (Brandenburg \& Putz, 2012) and SHELXTL (Sheldrick, 2008).

in DMF (20 mL) was added 1-bromononane (2.6 mmol). Stirring was continued at room temperature for $24 \mathrm{~h}$. The mixture was filtered and the solvent removed. The residue obtained was washed with water. The organic compound was chromatographed on a column of silica gel with ethyl acetatehexane (9/1) as eluent. Colourless crystals were isolated when the solvent was allowed to evaporate (yield $=79 \%$ ).

\section{Refinement}

Crystal data, data collection and structure refinement details are summarized in Table 5. The two carbon atoms at the end of the nonyl chain, C23 and C24, are disordered in a 0.562 (4)/ 0.438 (4) ratio. These were refined with restraints that the two components have comparable geometries. The $\mathrm{H}$ atoms on these carbons as well as those on $\mathrm{C} 22$ were included as riding contributions in idealized positions $(\mathrm{C}-\mathrm{H}=0.99 \AA$ with $U_{\text {iso }}(\mathrm{H})=1.5 U_{\text {eq }}(\mathrm{C})$.

\section{Funding information}

The support of NSF-MRI grant No. 1228232 for the purchase of the diffractometer and Tulane University for support of the Tulane Crystallography Laboratory are gratefully acknowledged. $\mathrm{TH}$ is grateful to Hacettepe University Scientific Research Project Unit (grant No. 013 D04 602 004). 


\section{References}

Armenise, D., Muraglia, M., Florio, M. A., Laurentis, N. D., Rosato, A., Carrieri, A., Corbo, F. \& Franchini, C. (2012). Mol. Pharmacol. Mol. Pharmacol, 50, 1178-1188.

Brandenburg, K. \& Putz, H. (2012). DIAMOND, Crystal Impact GbR, Bonn, Germany.

Bruker (2016). APEX3, SAINT and SADABS. Bruker AXS, Inc., Madison, Wisconsin, USA

Ellouz, M., Elmsellem, H., Sebbar, N. K., Steli, H., Al Mamari, K., Nadeem, A., Ouzidan, Y., Essassi, E. M., Abdel-Rahaman, I. \& Hristov, P. (2016b). J. Mater. Environ. Sci. 7, 2482-2497.

Ellouz, M., Sebbar, N. K., Boulhaoua, M., Essassi, E. M. \& Mague, J. T. (2017a). IUCr Data, 2, x170646.

Ellouz, M., Sebbar, N. K., Elmsellem, H., Steli, H., Fichtali, I., Mohamed, A. M. M., Mamari, K. A., Essassi, E. M. \& AbdelRahaman, I. (2016a). J. Mater. Environ. Sci. 7, 2806-2819.

Ellouz, M., Sebbar, N. K., Essassi, E. M., Ouzidan, Y., Mague, J. T. \& Zouihri, H. (2016c). IUCrData, 1, x160764.

Ellouz, M., Sebbar, N. K., Fichtali, I., Ouzidan, Y., Mennane, Z., Charof, R., Mague, J. T., Urrutigoïty, M. \& Essassi, E. M. (2018). Chem. Cent. J. 12, 123.

Ellouz, M., Sebbar, N. K., Ouzidan, Y., Essassi, E. M. \& Mague, J. T. (2017b). IUCr Data, 2, x170097.

Frisch, M. J., et al. (2009). GAUSSIAN09. Gaussian Inc., Wallingford, CT, USA.

Gowda, J., Khader, A. M. A., Kalluraya, B., Shree, P. \& Shabaraya, A. R. (2011). Eur. J. Med. Chem. 46, 4100.

Groom, C. R., Bruno, I. J., Lightfoot, M. P. \& Ward, S. C. (2016). Acta Cryst. B72, 171-179.

Gupta, R. R., Kumar, R. \& Gautam, R. K. (1985). J. Fluor. Chem. 28, 381-385.

Gupta, V. \& Gupta, R. R. (1991). J. Prakt. Chem. 333, 153-156.

Hathwar, V. R., Sist, M., Jørgensen, M. R. V., Mamakhel, A. H., Wang, X., Hoffmann, C. M., Sugimoto, K., Overgaard, J. \& Iversen, B. B. (2015). IUCrJ, 2, 563-574.

Hirshfeld, H. L. (1977). Theor. Chim. Acta, 44, 129-138.

Hni, B., Sebbar, N. K., Hökelek, T., El Ghayati, L., Bouzian, Y., Mague, J. T. \& Essassi, E. M. (2019b). Acta Cryst. E75, 593-599.

Hni, B., Sebbar, N. K., Hökelek, T., Ouzidan, Y., Moussaif, A., Mague, J. T. \& Essassi, E. M. (2019a). Acta Cryst. E75, 372-377.

Jayatilaka, D., Grimwood, D. J., Lee, A., Lemay, A., Russel, A. J., Taylor, C., Wolff, S. K., Cassam-Chenai, P. \& Whitton, A. (2005). TONTO - A System for Computational Chemistry. Available at: http://hirshfeldsurface.net/

Krause, L., Herbst-Irmer, R., Sheldrick, G. M. \& Stalke, D. (2015). J. Appl. Cryst. 48, 3-10.

Mackenzie, C. F., Spackman, P. R., Jayatilaka, D. \& Spackman, M. A. (2017). IUCrJ, 4, 575-587.
Malagu, K., Boustie, J., David, M., Sauleau, J., Amoros, M., Girre, R. L. \& Sauleau, A. (1998). Pharm. Pharmacol. Commun. 4, 57-60. McKinnon, J. J., Jayatilaka, D. \& Spackman, M. A. (2007). Chem. Commun. pp. 3814-3816.

Sabatini, S., Kaatz, G. W., Rossolini, G. M., Brandini, D. \& Fravolini, A. (2008). J. Med. Chem. 51, 4321-4330.

Sebbar, N. K., Ellouz, M., Essassi, E. M., Ouzidan, Y. \& Mague, J. T. (2015a). Acta Cryst. E71, o999.

Sebbar, N. K., Ellouz, M., Essassi, E. M., Saadi, M. \& El Ammari, L. (2015b). Acta Cryst. E71, o423-o424.

Sebbar, N. K., Ellouz, M., Essassi, E. M., Saadi, M. \& El Ammari, L. (2016a). IUCr Data.1, x161012.

Sebbar, N. K., Ellouz, M., Mague, J. T., Ouzidan, Y., Essassi, E. M. \& Zouihri, H. (2016c). IUCrData, 1, x160863.

Sebbar, N. K., Ellouz, M., Ouzidan, Y., Kaur, M., Essassi, E. M. \& Jasinski, J. P. (2017). IUCrData, 2, x170889.

Sebbar, N. K., Hni, B., Hökelek, T., Jaouhar, A., Labd Taha, M., Mague, J. T. \& Essassi, E. M. (2019a). Acta Cryst. E75, 721-727.

Sebbar, N. K., Hni, B., Hökelek, T., Labd Taha, M., Mague, J. T., El Ghayati, L. \& Essassi, E. M. (2019b). Acta Cryst. E75, 1650-1656.

Sebbar, N. K., Mekhzoum, M. E. M., Essassi, E. M., Zerzouf, A., Talbaoui, A., Bakri, Y., Saadi, M. \& Ammari, L. E. (2016b). Res. Chem. Intermed. 42, 6845-6862.

Sebbar, N. K., Zerzouf, A., Essassi, E. M., Saadi, M. \& El Ammari, L. (2014a). Acta Cryst. E70, o614.

Sheldrick, G. M. (2008). Acta Cryst. A64, 112-122.

Sheldrick, G. M. (2015a). Acta Cryst. A71, 3-8.

Sheldrick, G. M. (2015b). Acta Cryst. C71, 3-8.

Spackman, M. A. \& Jayatilaka, D. (2009). CrystEngComm, 11, 19-32.

Spackman, M. A., McKinnon, J. J. \& Jayatilaka, D. (2008). Cryst. Eng. Comm, 10, 377-388.

Tawada, H., Sugiyama, Y., Ikeda, H., Yamamoto, Y. \& Meguro, K. (1990). Chem. Pharm. Bull. 38, 1238-1245.

Trapani, G., Reho, A., Morlacchi, F., Latrofa, A., Marchini, P., Venturi, F. \& Cantalamessa, F. (1985). Farm. Ed. Sci. 40, 369-376.

Turner, M. J., Grabowsky, S., Jayatilaka, D. \& Spackman, M. A. (2014). J. Phys. Chem. Lett. 5, 4249-4255.

Turner, M. J., McKinnon, J. J., Wolff, S. K., Grimwood, D. J., Spackman, P. R., Jayatilaka, D. \& Spackman, M. A. (2017). CrystalExplorer17. The University of Western Australia.

Turner, M. J., Thomas, S. P., Shi, M. W., Jayatilaka, D. \& Spackman, M. A. (2015). Chem. Commun. 51, 3735-3738.

Venkatesan, P., Thamotharan, S., Ilangovan, A., Liang, H. \& Sundius, T. (2016). Spectrochim. Acta Part A, 153, 625-636.

Vidal, A., Madelmont, J. C. \& Mounetou, E. A. (2006). Synthesis, pp. 591-593.

Warren, B. K. \& Knaus, E. E. (1987). Eur. J. Med. Chem. 22, 411-415.

Zia-ur-Rehman, M., Choudary, J. A., Elsegood, M. R. J., Siddiqui, H. L. \& Khan, K. M. (2009). Eur. J. Med. Chem. 44, 1311-1316. 


\section{supporting information}

Acta Cryst. (2020). E76, 281-287 [https://doi.org/10.1107/S2056989020001036]

Crystal structure, Hirshfeld surface analysis, interaction energy and DFT studies of (2Z)-2-(2,4-dichlorobenzylidene)-4-nonyl-3,4-dihydro-2H-1,4-benzothiazin-3-one

Brahim Hni, Nada Kheira Sebbar, Tuncer Hökelek, Achour Redouane, Joel T. Mague, Noureddine Hamou Ahabchane and El Mokhtar Essassi

Computing details

Data collection: APEX3 (Bruker, 2016); cell refinement: SAINT (Bruker, 2016); data reduction: SAINT (Bruker, 2016); program(s) used to solve structure: SHELXT (Sheldrick, 2015a); program(s) used to refine structure: SHELXL2018/1 (Sheldrick, 2015b); molecular graphics: DIAMOND (Brandenburg \& Putz, 2012); software used to prepare material for publication: SHELXTL (Sheldrick, 2008).

(2Z)-2-(2,4-Dichlorobenzylidene)-4-nonyl-3,4-dihydro-2H-1,4-benzothiazin-3-one

Crystal data

$\mathrm{C}_{24} \mathrm{H}_{27} \mathrm{Cl}_{2} \mathrm{NOS}$

$M_{r}=448.42$

Triclinic, $P \overline{1}$

$a=8.9961(3) \AA$

$b=10.3755$ (3) $\AA$

$c=13.2565$ (4) $\AA$

$\alpha=73.857(1)^{\circ}$

$\beta=88.119(1)^{\circ}$

$\gamma=74.182(1)^{\circ}$

$V=1142.32(6) \AA^{3}$

Data collection

Bruker D8 VENTURE PHOTON 100 CMOS diffractometer

Radiation source: INCOATEC I $\mu \mathrm{S}$ micro-focus source

Mirror monochromator

Detector resolution: 10.4167 pixels $\mathrm{mm}^{-1}$

$\omega$ scans

Absorption correction: multi-scan

(SADABS; Krause et al., 2015)

Refinement

Refinement on $F^{2}$

Least-squares matrix: full

$R\left[F^{2}>2 \sigma\left(F^{2}\right)\right]=0.041$

$w R\left(F^{2}\right)=0.107$
$Z=2$

$F(000)=472$

$D_{\mathrm{x}}=1.304 \mathrm{Mg} \mathrm{m}^{-3}$

$\mathrm{Cu} K \alpha$ radiation, $\lambda=1.54178 \AA$

Cell parameters from 7317 reflections

$\theta=3.5-72.4^{\circ}$

$\mu=3.52 \mathrm{~mm}^{-1}$

$T=150 \mathrm{~K}$

Column, colourless

$0.20 \times 0.14 \times 0.08 \mathrm{~mm}$

$T_{\min }=0.54, T_{\max }=0.76$

8788 measured reflections

4246 independent reflections

3772 reflections with $I>2 \sigma(I)$

$R_{\text {int }}=0.025$

$\theta_{\text {max }}=72.4^{\circ}, \theta_{\text {min }}=3.5^{\circ}$

$h=-10 \rightarrow 11$

$k=-12 \rightarrow 12$

$l=-13 \rightarrow 15$

$S=1.01$

4246 reflections

349 parameters

14 restraints 
Primary atom site location: dual space

Secondary atom site location: difference Fourier map

Hydrogen site location: mixed

$\mathrm{H}$ atoms treated by a mixture of independent and constrained refinement

$$
\begin{aligned}
& w=1 /\left[\sigma^{2}\left(F_{\mathrm{o}}^{2}\right)+(0.0512 P)^{2}+0.6585 P\right] \\
& \text { where } P=\left(F_{\mathrm{o}}^{2}+2 F_{\mathrm{c}}^{2}\right) / 3 \\
& (\Delta / \sigma)_{\max }<0.001 \\
& \Delta \rho_{\max }=0.32 \mathrm{e} \AA^{-3} \\
& \Delta \rho_{\min }=-0.50 \mathrm{e} \AA^{-3}
\end{aligned}
$$

\section{Special details}

Geometry. All esds (except the esd in the dihedral angle between two 1.s. planes) are estimated using the full covariance matrix. The cell esds are taken into account individually in the estimation of esds in distances, angles and torsion angles; correlations between esds in cell parameters are only used when they are defined by crystal symmetry. An approximate (isotropic) treatment of cell esds is used for estimating esds involving 1.s. planes.

Refinement. Refinement of $\mathrm{F}^{2}$ against ALL reflections. The weighted R-factor $\mathrm{wR}$ and goodness of fit $\mathrm{S}$ are based on $\mathrm{F}^{2}$, conventional R-factors $R$ are based on $F$, with $F$ set to zero for negative $F^{2}$. The threshold expression of $F^{2}>2$ sigma $\left(F^{2}\right)$ is used only for calculating R-factors(gt) etc. and is not relevant to the choice of reflections for refinement. R-factors based on $\mathrm{F}^{2}$ are statistically about twice as large as those based on $\mathrm{F}$, and R- factors based on ALL data will be even larger. The

\begin{tabular}{|c|c|c|c|c|c|}
\hline & $x$ & $y$ & $z$ & $U_{\text {iso }} * / U_{\text {eq }}$ & Occ. $(<1)$ \\
\hline Cl1 & $0.49354(7)$ & $0.17182(5)$ & $0.85050(4)$ & $0.04871(16)$ & \\
\hline $\mathrm{Cl} 2$ & $0.90576(10)$ & $-0.32166(6)$ & $1.00981(5)$ & $0.0707(2)$ & \\
\hline $\mathrm{S} 1$ & $0.74621(5)$ & $0.09525(4)$ & $0.49738(4)$ & $0.03192(13)$ & \\
\hline $\mathrm{O} 1$ & $0.30841(14)$ & $0.26156(14)$ & $0.47052(11)$ & $0.0350(3)$ & \\
\hline N1 & $0.49478(16)$ & $0.35999(15)$ & $0.39383(12)$ & $0.0290(3)$ & \\
\hline $\mathrm{C} 1$ & 0.77339 (19) & $0.26057(18)$ & $0.43712(14)$ & $0.0278(4)$ & \\
\hline $\mathrm{C} 2$ & $0.9224(2)$ & $0.2768(2)$ & $0.43323(15)$ & $0.0330(4)$ & \\
\hline $\mathrm{H} 2$ & $1.005(3)$ & $0.196(3)$ & $0.4656(18)$ & $0.042(6)^{*}$ & \\
\hline $\mathrm{C} 3$ & $0.9469(2)$ & $0.4057(2)$ & $0.38810(17)$ & $0.0380(4)$ & \\
\hline $\mathrm{H} 3$ & $1.051(3)$ & $0.414(3)$ & $0.387(2)$ & $0.052(7)^{*}$ & \\
\hline $\mathrm{C} 4$ & $0.8221(2)$ & $0.5199(2)$ & $0.34844(18)$ & $0.0410(5)$ & \\
\hline $\mathrm{H} 4$ & $0.837(3)$ & $0.613(3)$ & $0.316(2)$ & $0.056(7)^{*}$ & \\
\hline $\mathrm{C} 5$ & $0.6730(2)$ & $0.5053(2)$ & $0.35211(17)$ & $0.0366(4)$ & \\
\hline $\mathrm{H} 5$ & $0.589(3)$ & $0.587(2)$ & $0.3270(18)$ & $0.039(6)^{*}$ & \\
\hline C6 & 0.64689 (19) & $0.37512(19)$ & $0.39486(14)$ & $0.0287(4)$ & \\
\hline $\mathrm{C} 7$ & $0.4449(2)$ & $0.26179(18)$ & $0.46805(14)$ & $0.0286(4)$ & \\
\hline $\mathrm{C} 8$ & $0.5633(2)$ & $0.15326(18)$ & $0.54591(15)$ & $0.0291(4)$ & \\
\hline C9 & 0.5205 & $0.1027(2)$ & $0.64295(16)$ & $0.0344(4)$ & \\
\hline $\mathrm{H} 9$ & $0.408(3)$ & $0.145(3)$ & $0.656(2)$ & $0.057(7)^{*}$ & \\
\hline $\mathrm{C} 10$ & $0.6193(2)$ & $-0.0020(2)$ & $0.73085(15)$ & $0.0331(4)$ & \\
\hline $\mathrm{C} 11$ & $0.6141(2)$ & $0.0188(2)$ & $0.83067(16)$ & $0.0361(4)$ & \\
\hline $\mathrm{C} 12$ & $0.7034(3)$ & $-0.0761(2)$ & $0.91616(17)$ & $0.0418(5)$ & \\
\hline H12 & $0.700(3)$ & -0.059 & $0.986(2)$ & $0.056(7)^{*}$ & \\
\hline $\mathrm{C} 13$ & $0.8000(3)$ & $-0.1984(2)$ & $0.90162(17)$ & $0.0429(5)$ & \\
\hline $\mathrm{C} 14$ & $0.8106(3)$ & $-0.2233(2)$ & $0.80462(17)$ & $0.0407(5)$ & \\
\hline H14 & $0.880(3)$ & -0.309 & $0.797(2)$ & $0.050(7)^{*}$ & \\
\hline $\mathrm{C} 15$ & $0.7214(2)$ & $-0.1252(2)$ & $0.72000(16)$ & $0.0363(4)$ & \\
\hline
\end{tabular}
two carbons at the end of the nonyl chain, C23 and C24, are disordered in a 0.562 (4)/0.438 (4) ratio. These were refined with restraints that the two components have comparable geometries. The H-atoms on these carbons as well as those on C22 were included as riding contributions in idealized positions.

Fractional atomic coordinates and isotropic or equivalent isotropic displacement parameters $\left(\AA^{2}\right)$ 


\begin{tabular}{|c|c|c|c|c|c|}
\hline H15 & $0.729(3)$ & $-0.149(3)$ & $0.655(2)$ & $0.046(6)^{*}$ & \\
\hline $\mathrm{C} 16$ & $0.3799(2)$ & $0.4592(2)$ & $0.31152(15)$ & $0.0309(4)$ & \\
\hline H16A & $0.315(3)$ & $0.405(2)$ & $0.2923(17)$ & $0.036(6)^{*}$ & \\
\hline H16B & $0.434(2)$ & $0.491(2)$ & $0.2491(17)$ & $0.028(5)^{*}$ & \\
\hline $\mathrm{C} 17$ & $0.2769(2)$ & $0.5837(2)$ & $0.34303(15)$ & $0.0313(4)$ & \\
\hline H17A & $0.225(3)$ & $0.548(2)$ & $0.4065(18)$ & $0.036(6)^{*}$ & \\
\hline H17B & $0.342(3)$ & $0.636(2)$ & $0.3630(17)$ & $0.032(5)^{*}$ & \\
\hline $\mathrm{C} 18$ & $0.1618(2)$ & $0.6778(2)$ & $0.25291(16)$ & $0.0325(4)$ & \\
\hline $\mathrm{H} 18 \mathrm{~A}$ & $0.104(3)$ & $0.623(2)$ & $0.2318(17)$ & $0.036(6)^{*}$ & \\
\hline H18B & $0.217(3)$ & $0.703(2)$ & $0.1916(19)$ & $0.040(6)^{*}$ & \\
\hline $\mathrm{C} 19$ & $0.0581(2)$ & $0.8062(2)$ & $0.27852(17)$ & $0.0348(4)$ & \\
\hline H19A & $0.004(3)$ & $0.776(2)$ & $0.3390(18)$ & $0.035(6)^{*}$ & \\
\hline H19B & $0.123(3)$ & $0.859(3)$ & $0.2959(19)$ & $0.045(6)^{*}$ & \\
\hline $\mathrm{C} 20$ & $-0.0531(2)$ & $0.9040(2)$ & $0.18839(17)$ & $0.0372(4)$ & \\
\hline $\mathrm{H} 20 \mathrm{~A}$ & $-0.125(3)$ & $0.853(2)$ & $0.1714(18)$ & $0.039(6)^{*}$ & \\
\hline $\mathrm{H} 20 \mathrm{~B}$ & $0.010(3)$ & $0.934(2)$ & $0.1228(19)$ & $0.043(6)^{*}$ & \\
\hline $\mathrm{C} 21$ & $-0.1479(2)$ & $1.0373(2)$ & $0.21183(18)$ & $0.0383(4)$ & \\
\hline $\mathrm{H} 21 \mathrm{~A}$ & $-0.205(3)$ & $1.011(3)$ & $0.274(2)$ & $0.059(8)^{*}$ & \\
\hline $\mathrm{H} 21 \mathrm{~B}$ & $-0.080(3)$ & $1.087(2)$ & $0.2266(18)$ & $0.042(6)^{*}$ & \\
\hline $\mathrm{C} 22$ & $-0.2613(3)$ & $1.1333(2)$ & 0.12285 (19) & $0.0493(6)$ & \\
\hline $\mathrm{H} 22 \mathrm{~A}$ & -0.325994 & 1.078902 & 0.104683 & $0.059^{*}$ & \\
\hline $\mathrm{H} 22 \mathrm{~B}$ & -0.201418 & 1.161858 & 0.060625 & $0.059^{*}$ & \\
\hline $\mathrm{C} 23$ & $-0.3697(8)$ & $1.2661(4)$ & $0.1422(6)$ & $0.0440(18)$ & $0.562(4)$ \\
\hline $\mathrm{H} 23 \mathrm{~A}$ & -0.458686 & 1.305117 & 0.090467 & $0.053^{*}$ & $0.562(4)$ \\
\hline $\mathrm{H} 23 \mathrm{~B}$ & -0.409306 & 1.245547 & 0.213838 & $0.053^{*}$ & $0.562(4)$ \\
\hline $\mathrm{C} 24$ & $-0.2717(6)$ & $1.3686(5)$ & $0.1297(4)$ & $0.0630(10)$ & $0.562(4)$ \\
\hline $\mathrm{H} 24 \mathrm{~A}$ & -0.335043 & 1.455992 & 0.141231 & $0.094^{*}$ & $0.562(4)$ \\
\hline $\mathrm{H} 24 \mathrm{~B}$ & -0.184022 & 1.328099 & 0.181244 & $0.094^{*}$ & $0.562(4)$ \\
\hline $\mathrm{H} 24 \mathrm{C}$ & -0.233125 & 1.387336 & 0.058565 & $0.094^{*}$ & $0.562(4)$ \\
\hline $\mathrm{C} 23 \mathrm{~A}$ & $-0.3282(12)$ & $1.2751(6)$ & $0.1453(7)$ & $0.0440(18)$ & $0.438(4)$ \\
\hline $\mathrm{H} 23 \mathrm{C}$ & -0.388048 & 1.262316 & 0.209642 & $0.053^{*}$ & $0.438(4)$ \\
\hline $\mathrm{H} 23 \mathrm{D}$ & -0.242430 & 1.312260 & 0.158121 & $0.053^{*}$ & $0.438(4)$ \\
\hline $\mathrm{C} 24 \mathrm{~A}$ & $-0.4333(8)$ & $1.3801(6)$ & $0.0534(5)$ & $0.0630(10)$ & $0.438(4)$ \\
\hline $\mathrm{H} 24 \mathrm{D}$ & -0.474134 & 1.469164 & 0.069938 & $0.094^{*}$ & $0.438(4)$ \\
\hline $\mathrm{H} 24 \mathrm{E}$ & -0.373809 & 1.394060 & -0.010101 & $0.094^{*}$ & $0.438(4)$ \\
\hline $\mathrm{H} 24 \mathrm{~F}$ & -0.519298 & 1.344161 & 0.041374 & $0.094 *$ & $0.438(4)$ \\
\hline
\end{tabular}

Atomic displacement parameters $\left(\AA^{2}\right)$

\begin{tabular}{lllllll}
\hline & $U^{11}$ & $U^{22}$ & $U^{33}$ & $U^{12}$ & $U^{13}$ & $U^{23}$ \\
\hline C11 & $0.0592(3)$ & $0.0364(3)$ & $0.0409(3)$ & $-0.0014(2)$ & $0.0109(2)$ & $-0.0080(2)$ \\
C12 & $0.1011(6)$ & $0.0441(3)$ & $0.0459(3)$ & $0.0100(3)$ & $-0.0229(3)$ & $-0.0052(2)$ \\
S1 & $0.0281(2)$ & $0.0251(2)$ & $0.0361(3)$ & $-0.00014(16)$ & $0.00197(17)$ & $-0.00517(17)$ \\
O1 & $0.0233(6)$ & $0.0393(7)$ & $0.0427(8)$ & $-0.0097(5)$ & $-0.0023(5)$ & $-0.0104(6)$ \\
N1 & $0.0200(7)$ & $0.0281(7)$ & $0.0351(8)$ & $-0.0032(6)$ & $-0.0043(6)$ & $-0.0054(6)$ \\
C1 & $0.0231(8)$ & $0.0284(8)$ & $0.0289(9)$ & $-0.0028(7)$ & $0.0005(6)$ & $-0.0074(7)$ \\
C2 & $0.0207(8)$ & $0.0363(10)$ & $0.0357(10)$ & $0.0002(7)$ & $-0.0005(7)$ & $-0.0076(8)$ \\
C3 & $0.0217(9)$ & $0.0421(11)$ & $0.0473(12)$ & $-0.0077(8)$ & $0.0014(8)$ & $-0.0088(9)$
\end{tabular}




$\begin{array}{lllllll}\text { C4 } & 0.0275(9) & 0.0339(10) & 0.0569(13) & -0.0089(8) & 0.0019(8) & -0.0049(9) \\ \text { C5 } & 0.0244(9) & 0.0276(9) & 0.0502(12) & -0.0032(7) & -0.0027(8) & -0.0020(8) \\ \text { C6 } & 0.0198(8) & 0.0299(9) & 0.0338(9) & -0.0038(7) & -0.0009(7) & -0.0076(7) \\ \text { C7 } & 0.0248(8) & 0.0282(8) & 0.0336(9) & -0.0058(7) & -0.0016(7) & -0.0111(7) \\ \text { C8 } & 0.0248(8) & 0.0264(8) & 0.0359(10) & -0.0063(7) & -0.0018(7) & -0.0087(7) \\ \text { C9 } & 0.0295(9) & 0.0343(10) & 0.0384(10) & -0.0093(8) & 0.0010(8) & -0.0081(8) \\ \text { C10 } & 0.0312(9) & 0.0335(9) & 0.0345(10) & -0.0129(8) & 0.0021(7) & -0.0055(8) \\ \text { C11 } & 0.0403(10) & 0.0294(9) & 0.0358(10) & -0.0091(8) & 0.0067(8) & -0.0057(8) \\ \text { C12 } & 0.0550(13) & 0.0372(11) & 0.0312(11) & -0.0117(9) & 0.0019(9) & -0.0077(8) \\ \text { C13 } & 0.0527(12) & 0.0326(10) & 0.0382(11) & -0.0078(9) & -0.0058(9) & -0.0045(8) \\ \text { C14 } & 0.0463(12) & 0.0320(10) & 0.0434(12) & -0.0083(9) & -0.0026(9) & -0.0117(8) \\ \text { C15 } & 0.0402(11) & 0.0361(10) & 0.0361(10) & -0.0141(8) & 0.0009(8) & -0.0122(8) \\ \text { C16 } & 0.0240(8) & 0.0312(9) & 0.0326(10) & -0.0018(7) & -0.0053(7) & -0.0058(7) \\ \text { C17 } & 0.0230(8) & 0.0306(9) & 0.0374(10) & -0.0033(7) & -0.0048(7) & -0.0080(8) \\ \text { C18 } & 0.0253(9) & 0.0322(9) & 0.0359(10) & -0.0033(7) & -0.0033(8) & -0.0067(8) \\ \text { C19 } & 0.0295(9) & 0.0320(10) & 0.0396(11) & -0.0032(8) & -0.0058(8) & -0.0089(8) \\ \text { C20 } & 0.0332(10) & 0.0318(10) & 0.0406(11) & 0.0006(8) & -0.0052(8) & -0.0088(8) \\ \text { C21 } & 0.0350(10) & 0.0321(10) & 0.0440(12) & -0.0017(8) & -0.0041(9) & -0.0113(8) \\ \text { C22 } & 0.0505(13) & 0.0366(11) & 0.0476(13) & 0.0079(10) & -0.0053(10) & -0.0095(9) \\ \text { C23 } & 0.033(4) & 0.0350(13) & 0.0547(15) & 0.0018(17) & 0.001(2) & -0.0088(11) \\ \text { C24 } & 0.064(2) & 0.0489(19) & 0.061(2) & 0.0064(17) & -0.0022(17) & -0.0126(16) \\ \text { C23A } & 0.033(4) & 0.0350(13) & 0.0547(15) & 0.0018(17) & 0.001(2) & -0.0088(11) \\ \text { C24A } & 0.064(2) & 0.0489(19) & 0.061(2) & 0.0064(17) & -0.0022(17) & -0.0126(16) \\ & & & & & & \end{array}$

Geometric parameters (A, $\stackrel{\circ}{)}$

\begin{tabular}{llll}
\hline $\mathrm{C} 11-\mathrm{C} 11$ & $1.744(2)$ & $\mathrm{C} 16-\mathrm{H} 16 \mathrm{~B}$ & $0.97(2)$ \\
$\mathrm{C} 2-\mathrm{C} 13$ & $1.733(2)$ & $\mathrm{C} 17-\mathrm{C} 18$ & $1.528(2)$ \\
$\mathrm{S} 1-\mathrm{C} 8$ & $1.7578(18)$ & $\mathrm{C} 17-\mathrm{H} 17 \mathrm{~A}$ & $0.98(2)$ \\
$\mathrm{S} 1-\mathrm{C} 1$ & $1.7589(18)$ & $\mathrm{C} 17-\mathrm{H} 17 \mathrm{~B}$ & $0.98(2)$ \\
$\mathrm{O} 1-\mathrm{C} 7$ & $1.228(2)$ & $\mathrm{C} 18-\mathrm{C} 19$ & $1.521(3)$ \\
$\mathrm{N} 1-\mathrm{C} 7$ & $1.368(2)$ & $\mathrm{C} 18-\mathrm{H} 18 \mathrm{~A}$ & $0.96(2)$ \\
$\mathrm{N} 1-\mathrm{C} 6$ & $1.420(2)$ & $\mathrm{C} 18-\mathrm{H} 18 \mathrm{~B}$ & $0.95(2)$ \\
$\mathrm{N} 1-\mathrm{C} 16$ & $1.479(2)$ & $\mathrm{C} 19-\mathrm{C} 20$ & $1.519(3)$ \\
$\mathrm{C} 1-\mathrm{C} 2$ & $1.393(3)$ & $\mathrm{C} 19-\mathrm{H} 19 \mathrm{~A}$ & $0.95(2)$ \\
$\mathrm{C} 1-\mathrm{C} 6$ & $1.398(2)$ & $\mathrm{C} 19-\mathrm{H} 19 \mathrm{~B}$ & $0.97(3)$ \\
$\mathrm{C} 2-\mathrm{C} 3$ & $1.379(3)$ & $\mathrm{C} 20-\mathrm{C} 21$ & $1.522(3)$ \\
$\mathrm{C} 2-\mathrm{H} 2$ & $0.96(2)$ & $\mathrm{C} 20-\mathrm{H} 20 \mathrm{~A}$ & $1.00(2)$ \\
$\mathrm{C} 3-\mathrm{C} 4$ & $1.382(3)$ & $\mathrm{C} 20-\mathrm{H} 20 \mathrm{~B}$ & $1.04(2)$ \\
$\mathrm{C} 3-\mathrm{H} 3$ & $0.96(3)$ & $\mathrm{C} 21-\mathrm{C} 22$ & $1.515(3)$ \\
$\mathrm{C} 4-\mathrm{C} 5$ & $1.388(3)$ & $\mathrm{C} 21-\mathrm{H} 21 \mathrm{~A}$ & $0.97(3)$ \\
$\mathrm{C} 4-\mathrm{H} 4$ & $0.99(3)$ & $\mathrm{C} 21-\mathrm{H} 21 \mathrm{~B}$ & $0.96(3)$ \\
$\mathrm{C} 5-\mathrm{C} 6$ & $1.392(3)$ & $\mathrm{C} 22-\mathrm{C} 23$ & $1.537(4)$ \\
$\mathrm{C} 5-\mathrm{H} 5$ & $0.96(2)$ & $\mathrm{C} 22-\mathrm{C} 23 \mathrm{~A}$ & $1.537(4)$ \\
$\mathrm{C} 7-\mathrm{C} 8$ & $1.497(2)$ & $\mathrm{C} 22-\mathrm{H} 22 \mathrm{~A}$ & 0.9900 \\
$\mathrm{C} 8-\mathrm{C} 9$ & $1.334(3)$ & $\mathrm{C} 22-\mathrm{H} 22 \mathrm{~B}$ & 0.9900 \\
$\mathrm{C} 9-\mathrm{C} 10$ & $1.471(3)$ & $\mathrm{C} 23-\mathrm{C} 24$ & $1.530(7)$ \\
$\mathrm{C} 9-\mathrm{H} 9$ & $1.02(3)$ & $\mathrm{C} 23-\mathrm{H} 23 \mathrm{~A}$ & 0.9900
\end{tabular}




\begin{tabular}{|c|c|c|c|}
\hline $\mathrm{C} 10-\mathrm{C} 11$ & $1.396(3)$ & $\mathrm{C} 23-\mathrm{H} 23 \mathrm{~B}$ & 0.9900 \\
\hline $\mathrm{C} 10-\mathrm{C} 15$ & $1.397(3)$ & $\mathrm{C} 24-\mathrm{H} 24 \mathrm{~A}$ & 0.9800 \\
\hline $\mathrm{C} 11-\mathrm{C} 12$ & $1.381(3)$ & $\mathrm{C} 24-\mathrm{H} 24 \mathrm{~B}$ & 0.9800 \\
\hline $\mathrm{C} 12-\mathrm{C} 13$ & $1.388(3)$ & $\mathrm{C} 24-\mathrm{H} 24 \mathrm{C}$ & 0.9800 \\
\hline $\mathrm{C} 12-\mathrm{H} 12$ & $0.99(3)$ & $\mathrm{C} 23 \mathrm{~A}-\mathrm{C} 24 \mathrm{~A}$ & $1.530(8)$ \\
\hline $\mathrm{C} 13-\mathrm{C} 14$ & $1.376(3)$ & $\mathrm{C} 23 \mathrm{~A}-\mathrm{H} 23 \mathrm{C}$ & 0.9900 \\
\hline $\mathrm{C} 14-\mathrm{C} 15$ & $1.385(3)$ & $\mathrm{C} 23 \mathrm{~A}-\mathrm{H} 23 \mathrm{D}$ & 0.9900 \\
\hline C14-H14 & $0.97(3)$ & $\mathrm{C} 24 \mathrm{~A}-\mathrm{H} 24 \mathrm{D}$ & 0.9800 \\
\hline C15-H15 & $0.96(3)$ & $\mathrm{C} 24 \mathrm{~A}-\mathrm{H} 24 \mathrm{E}$ & 0.9800 \\
\hline $\mathrm{C} 16-\mathrm{C} 17$ & $1.525(3)$ & $\mathrm{C} 24 \mathrm{~A}-\mathrm{H} 24 \mathrm{~F}$ & 0.9800 \\
\hline $\mathrm{C} 16-\mathrm{H} 16 \mathrm{~A}$ & $0.99(2)$ & & \\
\hline $\mathrm{C} 11 \cdots \mathrm{C} 5^{\mathrm{i}}$ & $3.634(2)$ & $\mathrm{C} 21 \cdots \mathrm{H} 24 \mathrm{~B}$ & 2.86 \\
\hline $\mathrm{C} 11 \cdots \mathrm{C} 12^{\mathrm{ii}}$ & $3.548(2)$ & $\mathrm{C} 24 \cdots \mathrm{H} 21 \mathrm{~B}$ & 2.91 \\
\hline $\mathrm{Cl1} \cdots \mathrm{H} 9$ & $2.82(3)$ & $\mathrm{C} 24 \mathrm{~A} \cdots \mathrm{H} 24 \mathrm{E}^{\mathrm{viii}}$ & 2.44 \\
\hline $\mathrm{C} 11 \cdots \mathrm{H} 5^{\mathrm{i}}$ & $2.86(2)$ & $\mathrm{C} 24 \mathrm{~A} \cdots \mathrm{H} 24 \mathrm{~F}^{\text {viii }}$ & 2.70 \\
\hline $\mathrm{C} 11 \cdots \mathrm{H} 12^{\mathrm{ii}}$ & $2.92(3)$ & $\mathrm{C} 24 \mathrm{~A} \cdots \mathrm{H} 24 \mathrm{D}^{\text {viii }}$ & 1.94 \\
\hline $\mathrm{Cl} 2 \cdots \mathrm{H} 20 \mathrm{~A}^{\mathrm{iii}}$ & $3.13(2)$ & $\mathrm{H} 3 \cdots H 17 \mathrm{~A}^{\mathrm{ix}}$ & $2.42(4)$ \\
\hline $\mathrm{Cl} 2 \cdots \mathrm{H} 24 \mathrm{C}^{\mathrm{i}}$ & 3.01 & H5 $\cdots$ H17B & $2.21(4)$ \\
\hline $\mathrm{S} 1 \cdots \mathrm{N} 1$ & $3.0439(16)$ & $\mathrm{H} 5 \cdots \mathrm{H} 16 \mathrm{~B}$ & $2.33(3)$ \\
\hline $\mathrm{S} 1 \cdots \mathrm{C} 15$ & $3.236(2)$ & $\mathrm{H} 12 \cdots \mathrm{H} 22 \mathrm{~A}^{\mathrm{iii}}$ & 2.37 \\
\hline $\mathrm{S} 1 \cdots \mathrm{H} 15$ & $2.84(3)$ & $\mathrm{H} 16 \mathrm{~A} \cdots \mathrm{H} 18 \mathrm{~A}$ & $2.47(3)$ \\
\hline $\mathrm{S} 1 \cdots \mathrm{H} 2^{\mathrm{iv}}$ & $3.15(3)$ & $\mathrm{H} 16 \mathrm{~B} \cdots \mathrm{H} 24 \mathrm{D}^{\mathrm{vii}}$ & 2.54 \\
\hline $\mathrm{O} 1 \cdots \mathrm{C} 3^{\mathrm{v}}$ & $3.268(2)$ & H16B $\cdots H 18 B$ & $2.46(3)$ \\
\hline $\mathrm{O} 1 \cdots \mathrm{C} 17$ & $3.238(2)$ & $\mathrm{H} 16 \mathrm{~B} \cdots \mathrm{H} 24 \mathrm{~A}^{\mathrm{vii}}$ & 2.49 \\
\hline $\mathrm{O} 1 \cdots \mathrm{C} 15^{\mathrm{vi}}$ & $3.270(2)$ & H17A $\cdots$ H19A & $2.59(3)$ \\
\hline $\mathrm{O} 1 \cdots \mathrm{H} 3^{\mathrm{v}}$ & $2.51(3)$ & H17B $\cdots H 19 B$ & $2.55(4)$ \\
\hline $\mathrm{O} 1 \cdots \mathrm{H} 16 \mathrm{~A}$ & $2.43(2)$ & H18B $\cdots H 20 B$ & $2.55(3)$ \\
\hline $\mathrm{O} 1 \cdots \mathrm{H} 17 \mathrm{~A}$ & $2.75(2)$ & $\mathrm{H} 19 \mathrm{~A} \cdots \mathrm{H} 21 \mathrm{~A}$ & $2.58(4)$ \\
\hline $\mathrm{O} 1 \cdots \mathrm{H} 9$ & $2.49(3)$ & H19B $\cdots H 21 B$ & $2.51(4)$ \\
\hline $\mathrm{O} 1 \cdots \mathrm{H} 15^{\mathrm{vi}}$ & $2.36(3)$ & $\mathrm{H} 20 \mathrm{~A} \cdots \mathrm{H} 22 \mathrm{~A}$ & 2.49 \\
\hline $\mathrm{C} 5 \cdots \mathrm{C} 17$ & $3.430(3)$ & H20B $\cdots H 22 B$ & 2.54 \\
\hline $\mathrm{C} 5 \cdots \mathrm{C} 24^{\mathrm{vii}}$ & 3.58 & $\mathrm{H} 21 \mathrm{~A} \cdots \mathrm{H} 23 \mathrm{~B}$ & 2.55 \\
\hline $\mathrm{C} 6 \cdots \mathrm{C} 24^{\mathrm{vii}}$ & 3.58 & $\mathrm{H} 21 \mathrm{~A} \cdots \mathrm{H} 23 \mathrm{C}$ & 2.60 \\
\hline $\mathrm{C} 24 \mathrm{~A} \cdots \mathrm{C} 24 \mathrm{~A}^{\mathrm{viii}}$ & 2.48 & $\mathrm{H} 21 \mathrm{~B} \cdots \mathrm{H} 24 \mathrm{~B}$ & 2.32 \\
\hline $\mathrm{C} 2 \cdots \mathrm{H} 19 \mathrm{~A}^{\mathrm{i}}$ & $2.98(2)$ & $\mathrm{H} 21 \mathrm{~B} \cdots \mathrm{H} 23 \mathrm{D}$ & 2.34 \\
\hline $\mathrm{C} 5 \cdots \mathrm{H} 24 \mathrm{~A}^{\mathrm{vii}}$ & 2.99 & $\mathrm{H} 22 \mathrm{~B} \cdots \mathrm{H} 24 \mathrm{C}$ & 2.27 \\
\hline $\mathrm{C} 5 \cdots \mathrm{H} 16 \mathrm{~B}$ & $2.64(2)$ & $\mathrm{H} 22 \mathrm{~B} \cdots \mathrm{H} 24 \mathrm{E}$ & 2.43 \\
\hline $\mathrm{C} 5 \cdots \mathrm{H} 17 \mathrm{~B}$ & $2.93(3)$ & $\mathrm{H} 24 \mathrm{D} \cdots \mathrm{C} 24 \mathrm{~A}^{\text {viii }}$ & 1.94 \\
\hline $\mathrm{C} 7 \cdots \mathrm{H} 15^{\mathrm{vi}}$ & $2.95(3)$ & $\mathrm{H} 24 \mathrm{D} \cdots \mathrm{H} 24 \mathrm{D}^{\mathrm{viii}}$ & 1.82 \\
\hline 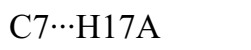 & $2.99(2)$ & $\mathrm{H} 24 \mathrm{D} \cdots \mathrm{H} 24 \mathrm{E}^{\text {viii }}$ & 1.70 \\
\hline $\mathrm{C} 16 \cdots \mathrm{H} 5$ & $2.62(3)$ & $\mathrm{H} 24 \mathrm{D} \cdots \mathrm{H} 24 \mathrm{~F}^{\mathrm{viii}}$ & 2.07 \\
\hline $\mathrm{C} 17 \cdots \mathrm{H} 3^{\mathrm{v}}$ & $2.98(3)$ & $\mathrm{H} 24 \mathrm{E} \cdots \mathrm{H} 24 \mathrm{~F}^{\mathrm{viii}}$ & 2.54 \\
\hline $\mathrm{C} 17 \cdots \mathrm{H} 5$ & $2.82(3)$ & & \\
\hline $\mathrm{C} 8-\mathrm{S} 1-\mathrm{C} 1$ & $97.27(8)$ & $\mathrm{C} 16-\mathrm{C} 17-\mathrm{H} 17 \mathrm{~B}$ & $109.2(13)$ \\
\hline $\mathrm{C} 7-\mathrm{N} 1-\mathrm{C} 6$ & $123.67(14)$ & $\mathrm{C} 18-\mathrm{C} 17-\mathrm{H} 17 \mathrm{~B}$ & $110.8(12)$ \\
\hline $\mathrm{C} 7-\mathrm{N} 1-\mathrm{C} 16$ & $117.19(14)$ & $\mathrm{H} 17 \mathrm{~A}-\mathrm{C} 17-\mathrm{H} 17 \mathrm{~B}$ & $106.0(18)$ \\
\hline
\end{tabular}




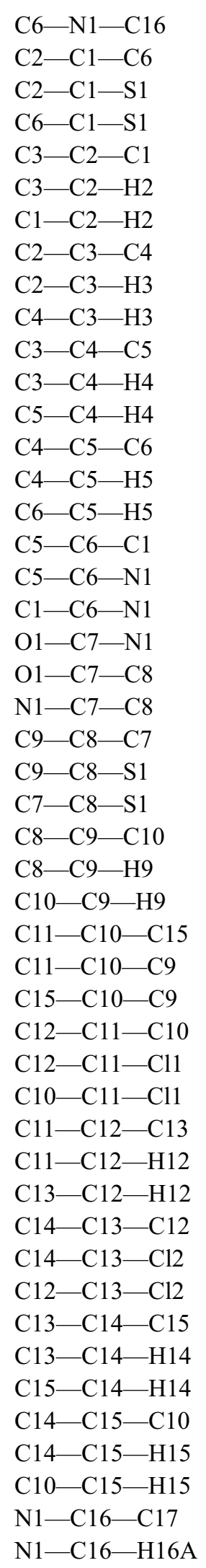

$119.07(15)$

$120.22(17)$

$119.25(13)$

$120.52(13)$

$120.53(17)$

$122.3(14)$

$117.1(14)$

$119.53(18)$

118.7 (16)

$121.7(16)$

$120.51(19)$

$120.8(16)$

$118.7(16)$

$120.58(17)$

118.4 (14)

$121.0(14)$

$118.59(16)$

$120.19(15)$

$121.22(16)$

$121.48(16)$

$121.01(16)$

$117.51(15)$

$118.51(17)$

125.47 (14)

$115.89(13)$

$126.86(18)$

$114.5(15)$

$118.6(15)$

$116.75(18)$

$120.39(18)$

$122.85(18)$

$122.93(19)$

$117.26(16)$

$119.80(15)$

$117.9(2)$

$121.9(16)$

$120.1(16)$

$121.42(19)$

$120.33(17)$

118.25 (17)

$119.3(2)$

$119.4(15)$

$121.3(15)$

121.63 (19)

$116.3(15)$

$121.9(15)$

$115.05(15)$

106.7 (13)
C19-C18-C17

C19-C18-H18A

C17-C18-H18A

C19-C18-H18B

C17-C18-H18B

$\mathrm{H} 18 \mathrm{~A}-\mathrm{C} 18-\mathrm{H} 18 \mathrm{~B}$

$\mathrm{C} 20-\mathrm{C} 19-\mathrm{C} 18$

$\mathrm{C} 20-\mathrm{C} 19-\mathrm{H} 19 \mathrm{~A}$

C18-C19-H19A

C20-C19-H19B

C18-C19-H19B

H19A-C19-H19B

C19-C20-C21

$\mathrm{C} 19-\mathrm{C} 20-\mathrm{H} 20 \mathrm{~A}$

$\mathrm{C} 21-\mathrm{C} 20-\mathrm{H} 20 \mathrm{~A}$

C19-C $20-\mathrm{H} 20 \mathrm{~B}$

$\mathrm{C} 21-\mathrm{C} 20-\mathrm{H} 20 \mathrm{~B}$

$\mathrm{H} 20 \mathrm{~A}-\mathrm{C} 20-\mathrm{H} 20 \mathrm{~B}$

$\mathrm{C} 22-\mathrm{C} 21-\mathrm{C} 20$

$\mathrm{C} 22-\mathrm{C} 21-\mathrm{H} 21 \mathrm{~A}$

$\mathrm{C} 20-\mathrm{C} 21-\mathrm{H} 21 \mathrm{~A}$

$\mathrm{C} 22-\mathrm{C} 21-\mathrm{H} 21 \mathrm{~B}$

$\mathrm{C} 20-\mathrm{C} 21-\mathrm{H} 21 \mathrm{~B}$

$\mathrm{H} 21 \mathrm{~A}-\mathrm{C} 21-\mathrm{H} 21 \mathrm{~B}$

$\mathrm{C} 21-\mathrm{C} 22-\mathrm{C} 23$

$\mathrm{C} 21-\mathrm{C} 22-\mathrm{C} 23 \mathrm{~A}$

$\mathrm{C} 21-\mathrm{C} 22-\mathrm{H} 22 \mathrm{~A}$

$\mathrm{C} 23-\mathrm{C} 22-\mathrm{H} 22 \mathrm{~A}$

$\mathrm{C} 21-\mathrm{C} 22-\mathrm{H} 22 \mathrm{~B}$

$\mathrm{C} 23-\mathrm{C} 22-\mathrm{H} 22 \mathrm{~B}$

$\mathrm{H} 22 \mathrm{~A}-\mathrm{C} 22-\mathrm{H} 22 \mathrm{~B}$

$\mathrm{C} 24-\mathrm{C} 23-\mathrm{C} 22$

$\mathrm{C} 24-\mathrm{C} 23-\mathrm{H} 23 \mathrm{~A}$

$\mathrm{C} 22-\mathrm{C} 23-\mathrm{H} 23 \mathrm{~A}$

$\mathrm{C} 24-\mathrm{C} 23-\mathrm{H} 23 \mathrm{~B}$

$\mathrm{C} 22-\mathrm{C} 23-\mathrm{H} 23 \mathrm{~B}$

$\mathrm{H} 23 \mathrm{~A}-\mathrm{C} 23-\mathrm{H} 23 \mathrm{~B}$

$\mathrm{C} 23-\mathrm{C} 24-\mathrm{H} 24 \mathrm{~A}$

$\mathrm{C} 23-\mathrm{C} 24-\mathrm{H} 24 \mathrm{~B}$

$\mathrm{H} 24 \mathrm{~A}-\mathrm{C} 24-\mathrm{H} 24 \mathrm{~B}$

$\mathrm{C} 23-\mathrm{C} 24-\mathrm{H} 24 \mathrm{C}$

$\mathrm{H} 24 \mathrm{~A}-\mathrm{C} 24-\mathrm{H} 24 \mathrm{C}$

$\mathrm{H} 24 \mathrm{~B}-\mathrm{C} 24-\mathrm{H} 24 \mathrm{C}$

$\mathrm{C} 24 \mathrm{~A}-\mathrm{C} 23 \mathrm{~A}-\mathrm{C} 22$

$\mathrm{C} 24 \mathrm{~A}-\mathrm{C} 23 \mathrm{~A}-\mathrm{H} 23 \mathrm{C}$

$\mathrm{C} 22-\mathrm{C} 23 \mathrm{~A}-\mathrm{H} 23 \mathrm{C}$

$\mathrm{C} 24 \mathrm{~A}-\mathrm{C} 23 \mathrm{~A}-\mathrm{H} 23 \mathrm{D}$

$\mathrm{C} 22-\mathrm{C} 23 \mathrm{~A}-\mathrm{H} 23 \mathrm{D}$
$113.07(16)$

$112.5(13)$

$109.3(13)$

$111.2(14)$

108.8 (14)

$101.4(19)$

$113.91(17)$

110.8 (14)

$108.3(14)$

$107.2(14)$

$108.7(15)$

107.7 (19)

$113.55(18)$

$108.8(13)$

$109.2(13)$

109.1 (13)

107.1 (13)

$109.1(19)$

$113.53(18)$

$108.6(17)$

$107.9(17)$

$108.5(14)$

$109.6(14)$

109 (2)

117.2 (4)

$109.3(4)$

108.0

108.0

108.0

108.0

107.2

105.7 (4)

110.6

110.6

110.6

110.6

108.7

109.5

109.5

109.5

109.5

109.5

109.5

111.3 (6)

109.4

109.4

109.4

109.4 


\begin{tabular}{|c|c|c|c|}
\hline $\mathrm{C} 17-\mathrm{C} 16-\mathrm{H} 16 \mathrm{~A}$ & $109.8(13)$ & $\mathrm{H} 23 \mathrm{C}-\mathrm{C} 23 \mathrm{~A}-\mathrm{H} 23 \mathrm{D}$ & 108.0 \\
\hline $\mathrm{N} 1-\mathrm{C} 16-\mathrm{H} 16 \mathrm{~B}$ & $108.8(12)$ & $\mathrm{C} 23 \mathrm{~A}-\mathrm{C} 24 \mathrm{~A}-\mathrm{H} 24 \mathrm{D}$ & 109.5 \\
\hline $\mathrm{C} 17-\mathrm{C} 16-\mathrm{H} 16 \mathrm{~B}$ & $110.0(12)$ & $\mathrm{C} 23 \mathrm{~A}-\mathrm{C} 24 \mathrm{~A}-\mathrm{H} 24 \mathrm{E}$ & 109.5 \\
\hline $\mathrm{H} 16 \mathrm{~A}-\mathrm{C} 16-\mathrm{H} 16 \mathrm{~B}$ & $106.0(18)$ & $\mathrm{H} 24 \mathrm{D}-\mathrm{C} 24 \mathrm{~A}-\mathrm{H} 24 \mathrm{E}$ & 109.5 \\
\hline $\mathrm{C} 16-\mathrm{C} 17-\mathrm{C} 18$ & $110.52(16)$ & $\mathrm{C} 23 \mathrm{~A}-\mathrm{C} 24 \mathrm{~A}-\mathrm{H} 24 \mathrm{~F}$ & 109.5 \\
\hline $\mathrm{C} 16-\mathrm{C} 17-\mathrm{H} 17 \mathrm{~A}$ & $107.9(13)$ & $\mathrm{H} 24 \mathrm{D}-\mathrm{C} 24 \mathrm{~A}-\mathrm{H} 24 \mathrm{~F}$ & 109.5 \\
\hline $\mathrm{C} 18-\mathrm{C} 17-\mathrm{H} 17 \mathrm{~A}$ & $112.2(13)$ & $\mathrm{H} 24 \mathrm{E}-\mathrm{C} 24 \mathrm{~A}-\mathrm{H} 24 \mathrm{~F}$ & 109.5 \\
\hline $\mathrm{C} 8-\mathrm{S} 1-\mathrm{C} 1-\mathrm{C} 2$ & $147.58(16)$ & $\mathrm{S} 1-\mathrm{C} 8-\mathrm{C} 9-\mathrm{C} 10$ & $5.5(3)$ \\
\hline $\mathrm{C} 8-\mathrm{S} 1-\mathrm{C} 1-\mathrm{C} 6$ & $-31.51(17)$ & $\mathrm{C} 8-\mathrm{C} 9-\mathrm{C} 10-\mathrm{C} 11$ & $133.9(2)$ \\
\hline $\mathrm{C} 6-\mathrm{C} 1-\mathrm{C} 2-\mathrm{C} 3$ & $0.2(3)$ & $\mathrm{C} 8-\mathrm{C} 9-\mathrm{C} 10-\mathrm{C} 15$ & $-46.2(3)$ \\
\hline $\mathrm{S} 1-\mathrm{C} 1-\mathrm{C} 2-\mathrm{C} 3$ & $-178.87(16)$ & $\mathrm{C} 15-\mathrm{C} 10-\mathrm{C} 11-\mathrm{C} 12$ & $-0.3(3)$ \\
\hline $\mathrm{C} 1-\mathrm{C} 2-\mathrm{C} 3-\mathrm{C} 4$ & $1.3(3)$ & $\mathrm{C} 9-\mathrm{C} 10-\mathrm{C} 11-\mathrm{C} 12$ & $179.60(19)$ \\
\hline $\mathrm{C} 2-\mathrm{C} 3-\mathrm{C} 4-\mathrm{C} 5$ & $-1.1(3)$ & $\mathrm{C} 15-\mathrm{C} 10-\mathrm{C} 11-\mathrm{C} 11$ & $178.39(14)$ \\
\hline $\mathrm{C} 3-\mathrm{C} 4-\mathrm{C} 5-\mathrm{C} 6$ & $-0.6(4)$ & $\mathrm{C} 9-\mathrm{C} 10-\mathrm{C} 11-\mathrm{Cl} 1$ & $-1.7(3)$ \\
\hline $\mathrm{C} 4-\mathrm{C} 5-\mathrm{C} 6-\mathrm{C} 1$ & $2.1(3)$ & $\mathrm{C} 10-\mathrm{C} 11-\mathrm{C} 12-\mathrm{C} 13$ & $-1.3(3)$ \\
\hline $\mathrm{C} 4-\mathrm{C} 5-\mathrm{C} 6-\mathrm{N} 1$ & $-177.04(19)$ & $\mathrm{C} 11-\mathrm{C} 11-\mathrm{C} 12-\mathrm{C} 13$ & $179.99(17)$ \\
\hline $\mathrm{C} 2-\mathrm{C} 1-\mathrm{C} 6-\mathrm{C} 5$ & $-1.9(3)$ & $\mathrm{C} 11-\mathrm{C} 12-\mathrm{C} 13-\mathrm{C} 14$ & $1.9(3)$ \\
\hline $\mathrm{S} 1-\mathrm{C} 1-\mathrm{C} 6-\mathrm{C} 5$ & $177.16(15)$ & $\mathrm{C} 11-\mathrm{C} 12-\mathrm{C} 13-\mathrm{C} 2$ & $-177.07(17)$ \\
\hline $\mathrm{C} 2-\mathrm{C} 1-\mathrm{C} 6-\mathrm{N} 1$ & $177.25(17)$ & $\mathrm{C} 12-\mathrm{C} 13-\mathrm{C} 14-\mathrm{C} 15$ & $-1.0(3)$ \\
\hline $\mathrm{S} 1-\mathrm{C} 1-\mathrm{C} 6-\mathrm{N} 1$ & $-3.7(2)$ & $\mathrm{Cl} 2-\mathrm{C} 13-\mathrm{C} 14-\mathrm{C} 15$ & $178.02(17)$ \\
\hline $\mathrm{C} 7-\mathrm{N} 1-\mathrm{C} 6-\mathrm{C} 5$ & $-150.53(19)$ & $\mathrm{C} 13-\mathrm{C} 14-\mathrm{C} 15-\mathrm{C} 10$ & $-0.7(3)$ \\
\hline $\mathrm{C} 16-\mathrm{N} 1-\mathrm{C} 6-\mathrm{C} 5$ & $26.4(3)$ & $\mathrm{C} 11-\mathrm{C} 10-\mathrm{C} 15-\mathrm{C} 14$ & $1.3(3)$ \\
\hline $\mathrm{C} 7-\mathrm{N} 1-\mathrm{C} 6-\mathrm{C} 1$ & $30.3(3)$ & $\mathrm{C} 9-\mathrm{C} 10-\mathrm{C} 15-\mathrm{C} 14$ & $-178.57(19)$ \\
\hline $\mathrm{C} 16-\mathrm{N} 1-\mathrm{C} 6-\mathrm{C} 1$ & $-152.76(17)$ & $\mathrm{C} 7-\mathrm{N} 1-\mathrm{C} 16-\mathrm{C} 17$ & $82.7(2)$ \\
\hline $\mathrm{C} 6-\mathrm{N} 1-\mathrm{C} 7-\mathrm{O} 1$ & $172.26(17)$ & $\mathrm{C} 6-\mathrm{N} 1-\mathrm{C} 16-\mathrm{C} 17$ & $-94.4(2)$ \\
\hline $\mathrm{C} 16-\mathrm{N} 1-\mathrm{C} 7-\mathrm{O} 1$ & $-4.7(3)$ & $\mathrm{N} 1-\mathrm{C} 16-\mathrm{C} 17-\mathrm{C} 18$ & $-179.23(15)$ \\
\hline $\mathrm{C} 6-\mathrm{N} 1-\mathrm{C} 7-\mathrm{C} 8$ & $-8.3(3)$ & $\mathrm{C} 16-\mathrm{C} 17-\mathrm{C} 18-\mathrm{C} 19$ & $-178.51(17)$ \\
\hline $\mathrm{C} 16-\mathrm{N} 1-\mathrm{C} 7-\mathrm{C} 8$ & $174.73(16)$ & $\mathrm{C} 17-\mathrm{C} 18-\mathrm{C} 19-\mathrm{C} 20$ & $177.77(17)$ \\
\hline $\mathrm{O} 1-\mathrm{C} 7-\mathrm{C} 8-\mathrm{C} 9$ & $-32.8(3)$ & $\mathrm{C} 18-\mathrm{C} 19-\mathrm{C} 20-\mathrm{C} 21$ & $-175.70(18)$ \\
\hline $\mathrm{N} 1-\mathrm{C} 7-\mathrm{C} 8-\mathrm{C} 9$ & $147.71(18)$ & $\mathrm{C} 19-\mathrm{C} 20-\mathrm{C} 21-\mathrm{C} 22$ & $-178.7(2)$ \\
\hline $\mathrm{O} 1-\mathrm{C} 7-\mathrm{C} 8-\mathrm{S} 1$ & $143.15(15)$ & $\mathrm{C} 20-\mathrm{C} 21-\mathrm{C} 22-\mathrm{C} 23$ & $176.0(3)$ \\
\hline $\mathrm{N} 1-\mathrm{C} 7-\mathrm{C} 8-\mathrm{S} 1$ & $-36.3(2)$ & $\mathrm{C} 20-\mathrm{C} 21-\mathrm{C} 22-\mathrm{C} 23 \mathrm{~A}$ & $-169.4(5)$ \\
\hline $\mathrm{C} 1-\mathrm{S} 1-\mathrm{C} 8-\mathrm{C} 9$ & $-133.86(18)$ & $\mathrm{C} 21-\mathrm{C} 22-\mathrm{C} 23-\mathrm{C} 24$ & $78.1(5)$ \\
\hline $\mathrm{C} 1-\mathrm{S} 1-\mathrm{C} 8-\mathrm{C} 7$ & $50.47(14)$ & $\mathrm{C} 21-\mathrm{C} 22-\mathrm{C} 23 \mathrm{~A}-\mathrm{C} 24 \mathrm{~A}$ & $175.2(6)$ \\
\hline $\mathrm{C} 7-\mathrm{C} 8-\mathrm{C} 9-\mathrm{C} 10$ & $-178.89(17)$ & & \\
\hline
\end{tabular}

Symmetry codes: (i) $-x+1,-y+1,-z+1$; (ii) $-x+1,-y,-z+2$; (iii) $x+1, y-1, z+1$; (iv) $-x+2,-y,-z+1$; (v) $x-1, y, z$; (vi) $-x+1,-y,-z+1$; (vii) $x+1, y-1, z$; (viii) $-x-1,-y+3,-z$; (ix) $x+1, y, z$.

Hydrogen-bond geometry $\left(A,{ }^{\circ}\right)$

$\mathrm{Cg} 1$ is the centroid of the ring $A(\mathrm{C} 1-\mathrm{C} 6)$.

\begin{tabular}{lllll}
\hline$D-\mathrm{H} \cdots A$ & $D-\mathrm{H}$ & $\mathrm{H} \cdots A$ & $D \cdots A$ & $D-\mathrm{H} \cdots A$ \\
\hline $\mathrm{C} 3-\mathrm{H} 3 \cdots \mathrm{O} 1^{\mathrm{ix}}$ & $0.96(3)$ & $2.51(3)$ & $3.268(2)$ & $136(2)$ \\
$\mathrm{C} 5-\mathrm{H} 5 \cdots \mathrm{Cl}^{\mathrm{i}}$ & $0.96(2)$ & $2.86(2)$ & $3.634(2)$ & $138.8(17)$
\end{tabular}


supporting information

$\begin{array}{lllll}\mathrm{C} 15-\mathrm{H} 15 \cdots \mathrm{O} 1^{\mathrm{vi}} & 0.96(3) & 2.36(3) & 3.270(2) & 159(2) \\ \mathrm{C} 17-\mathrm{H} 17 A^{\cdots} \mathrm{Cg} 1^{\mathrm{i}} & 0.98(2) & 2.90(2) & 3.619(2) & 131.2(17)\end{array}$

Symmetry codes: (i) $-x+1,-y+1,-z+1$; (vi) $-x+1,-y,-z+1$; (ix) $x+1, y, z$. 